Portland State University

PDXScholar

\title{
Anisotropy of the Reynolds Stress Tensor in the Wakes of Wind turbine Arrays in Cartesian Arrangements with Counter-Rotating Rotors
}

Nicholas Hamilton

Portland State University

Raúl Bayoán Cal

Portland State University

Follow this and additional works at: https://pdxscholar.library.pdx.edu/mengin_fac

Part of the Materials Science and Engineering Commons, and the Mechanical Engineering Commons Let us know how access to this document benefits you.

\section{Citation Details}

Hamilton, N., \& Cal, R. B. (2015). Anisotropy of the Reynolds stress tensor in the wakes of wind turbine arrays in Cartesian arrangements with counter-rotating rotors. Physics of Fluids (1994-present), 27(1), 015102.

This Article is brought to you for free and open access. It has been accepted for inclusion in Mechanical and Materials Engineering Faculty Publications and Presentations by an authorized administrator of PDXScholar. Please contact us if we can make this document more accessible: pdxscholar@pdx.edu. 


\title{
Anisotropy of the Reynolds stress tensor in the wakes of wind turbine arrays in Cartesian arrangements with counter-rotating rotors
}

\author{
Nicholas Hamilton ${ }^{a)}$ and Raúl Bayoán Cal ${ }^{\text {b) }}$ \\ Portland State University, 1930 SW 4th Avenue, Portland, Oregon 97215, USA
}

(Received 6 August 2014; accepted 28 November 2014; published online 9 January 2015)

\begin{abstract}
A $4 \times 3$ wind turbine array in a Cartesian arrangement was constructed in a wind tunnel setting with four configurations based on the rotational sense of the rotor blades. The fourth row of devices is considered to be in the fully developed turbine canopy for a Cartesian arrangement. Measurements of the flow field were made with stereo particle-image velocimetry immediately upstream and downstream of the selected model turbines. Rotational sense of the turbine blades is evident in the mean spanwise velocity $W$ and the Reynolds shear stress $-\overline{v w}$. The flux of kinetic energy is shown to be of greater magnitude following turbines in arrays where direction of rotation of the blades varies. Invariants of the normalized Reynolds stress anisotropy tensor $(\eta$ and $\xi$ ) are plotted in the Lumley triangle and indicate that distinct characters of turbulence exist in regions of the wake following the nacelle and the rotor blade tips. Eigendecomposition of the tensor yields principle components and corresponding coordinate system transformations. Characteristic spheroids representing the balance of components in the normalized anisotropy tensor are composed with the eigenvalues yielding shapes predicted by the Lumley triangle. Rotation of the coordinate system defined by the eigenvectors demonstrates trends in the streamwise coordinate following the rotors, especially trailing the top-tip of the rotor and below the hub. Direction of rotation of rotor blades is shown by the orientation of characteristic spheroids according to principle axes. In the inflows of exit row turbines, the normalized Reynolds stress anisotropy tensor shows cumulative effects of the upstream turbines, tending toward prolate shapes for uniform rotational sense, oblate spheroids for streamwise organization of rotational senses, and a mixture of characteristic shapes when the rotation varies by row. Comparison between the invariants of the Reynolds stress anisotropy tensor and terms from the mean mechanical energy equation indicate correlation between the degree of anisotropy and the regions of the wind turbine wakes where turbulence kinetic energy is produced. The flux of kinetic energy into the momentum-deficit area of the wake from above the canopy is associated with prolate characteristic spheroids. Flux upward into the wake from below the rotor area is associated with oblate characteristic spheroids. Turbulence in the region of the flow directly following the nacelle of the wind turbines demonstrates greater isotropy than regions following the rotor blades. The power and power coefficients for wind turbines indicate that flow structures on the order of magnitude of the spanwise turbine spacing that increase turbine efficiency depending on particular array configuration. (C) 2015 AIP Publishing LLC. [http://dx.doi.org/10.1063/1.4903968]
\end{abstract}

a) hamilton@ cecs.pdx.edu
b) cal@me.pdx.edu 


\section{INTRODUCTION}

Interest in large turbine arrays has increased as wind power becomes an attractive means of meeting energy needs although characterizing the turbine canopy layer remains a complex challenge. Energy production by wind turbines in large arrays has been correlated to the difference in the flux of mean kinetic energy into the wake, ${ }^{1}$ leading to the global entrainment of kinetic energy downward from above into the main canopy layer. Previous work has shown that energy-containing scales of turbulence associated with the top-tip of the rotor blades are responsible for much of the entrainment of energy into the canopy layer. ${ }^{2}$

It is common for wind farms to be designed through simulations as wind atlases (e.g., GIS raster layers) are available for many parts of the world. Numerical simulations of wind arrays have become larger and more complex, ${ }^{3-5}$ where many models use self-similar velocity profiles from experimental and theoretical work. The growth rate of wakes in turbine arrays is generally attributed to the level of turbulence encountered amplified by the mean shear in the wind turbine canopy. ${ }^{6}$ Often, the momentum deficit predicted in wind turbine wakes is obtained from global conservation principles. In many simulations, large wind farms are considered as a series of drag elements that increase the effective surface roughness for the atmospheric boundary layer (ABL) and decrease mean wind speeds near the ground. The optimization undertaken by Meyers and Meneveau ${ }^{7}$ addresses the turbine spacing in wind farms, where optimal spacing is a product of economical constraints and performance of turbines within the array. It was found that optimal spacing for turbines based on performance may be as large as 15 rotor diameters in the streamwise direction rather than the 6 or 7 diameters typically found in real installations. The simulation undertaken by Meyers and Meneveau makes use of a periodic boundary condition in the streamwise direction, leading to the "infinite" wind turbine array condition. For real installations or experiments, arrays may be large but cannot be considered infinite and the ideal spacing between devices may differ from the results presented in simulations of this type. Further optimization of arrays indicates that transverse staggering of devices is important to power output. Recent studies by Porté-Agel et al. ${ }^{8}$ and Stevens et al. ${ }^{9}$ found that the higher average power output for turbines in large arrays is obtained for a staggered wind-farm arrangements over Cartesian arrangements. The specific alignment angles between devices depend on spacing in both studies but the optimal designs maximize streamwise spacing and increase wake recovery within the arrays.

Wind turbine arrays can be treated as canopies ${ }^{10,11}$ in the ABL where transport processes differ from those of surface layer flows. Additionally, fluid stresses are expected exhibit anisotropy due to the swirl imparted to the flow from the passage of the rotor blades. ${ }^{12}$ To reproduce this effect, sophisticated simulations may opt for a computationally expensive model. Wind farms are being investigated through large eddy simulations ${ }^{7,13}$ for which terrains and boundary conditions are often idealized. Models balancing the transport equations of the Reynolds stresses are far more effective at representing the anisotropy of turbulence than the more computationally efficient $k-\epsilon$ or $k-\omega$ models. ${ }^{14}$

Although not commonly used in wind turbine array simulations, return to isotropy models, introduced by Rotta, ${ }^{15}$ have been employed to represent the asymptotic behavior of the stresses in the inner boundary layer. Explicit algebraic models are fairly common in second-order closure schemes of some Reynolds-averaged Navier-Stokes (RANS) models. ${ }^{16}$ Development and significance of the normalized Reynolds stress anisotropy tensor is detailed by Simonsen and Krogstad. ${ }^{17}$ Here, the principle components resulting from an eigenvalue decomposition were related to spheroidal structures characterizing the anisotropy of turbulence. Vectors resulting from eigendecomposition of the normalized anisotropy tensor form an orthonormal basis describing a coordinate system corresponding to the eigenvalues rotated from the original Cartesian system. The anisotropy invariant map (AIM) shows the span of realizable forms of anisotropic turbulence including special cases and theoretical limits. Each idealized form of turbulence is related to characteristic spheroids formed by the eigenvalues of the normalized Reynolds stress anisotropy tensor further explained in Sec. II.

Investigating the anisotropy of turbulence relating to turbomachinery, Tedds et al. ${ }^{18}$ mapped the wakes of tidal stream turbines, structurally similar to horizontal axis wind turbines, using acoustic 
Doppler velocimetry measurements. The normalized Reynolds stress anisotropy tensor was then decomposed according to the procedure outlined by Choi and Lumley ${ }^{19}$ and the resulting invariants plotted in the AIM (also referred to as the Lumley triangle). A tendency of characteristic shapes of turbulence toward oblate spheroids in far wake was demonstrated.

Investigating a flat-plate boundary layer, it was found by Mestayer ${ }^{20}$ that there is consistent evidence that local isotropy exists at scales smaller than twenty times the Kolmogorov microscale. Local isotropy is expected in the dissipative range of turbulent scales while larger scales demonstrate a higher degree of anisotropy. Smalley et al. ${ }^{21}$ showed that surface characteristics of the wall influence the balance of turbulent stresses. Their findings show normal stresses are more isotropic over the rough surfaces than on a smooth wall. Later work by Leonardi et al. ${ }^{22}$ confirmed through direct numerical simulations that the Reynolds stress anisotropy tensor and its invariants approach isotropic conditions with increased surface roughness.

Smyth and Moum ${ }^{23}$ determined the form of the Reynolds stress tensor for atmospheric flows finding that anisotropy in large-scale turbulence generates Reynolds stresses which extract energy from the background flow. Many results focus on shear layers analogous to flow on the border of a wind turbine wake and the ABL. Notable computational work regarding the anisotropy of turbulence in the wakes of wind turbines is presented by Gómez et al. ${ }^{16}$ and Jiménez et al. ${ }^{24}$ Both simulations employ a second-order closure model with explicit algebraic models for the components of the turbulent stress tensor.

A number of studies have been conducted regarding the rotational sense of horizontal axis wind turbines. The work by Ushiyama et $a l .^{25}$ consisted of a wind tunnel experiment wherein a counter-rotating device was constructed and tested, demonstrating the technical possibility of counter-rotating type wind powered generators. Simulation regarding the relative size and optimum placement of a two-stage wind turbine ${ }^{26}$ where the rotors were investigated through momentum theory for an experimental wake model. Similarly, simulations of the performance of a wind turbine with a pair of counter-rotating rotors have been undertaken by Lee et al. ${ }^{27}$ and Shen et al. ${ }^{28}$ finding that power production can be increased on the order of 10\%-20\% as compared to a wind turbine with a single rotor. Recent work by Yuan et al. ${ }^{29}$ detailed the performance of counter-rotating versus co-rotating devices finding that significant azimuthal velocity in the wakes is of benefit to the production of successive devices, but that the benefit decreases sharply as a function of spacing. It was also found that counter-rotating turbine blades reduce the fatigue loads experienced by the blades, leading to longer material lifetimes.

The current work compares the turbulence in measurement planes directly upstream and downstream of wind turbines in various configurations of an array in which the rotational sense of the rotors is varied. The Reynolds stress anisotropy tensor is decomposed into its principle components and a related coordinate system. Invariants of the anisotropy tensor are plotted on the Lumley triangle in order to assess the state of turbulence in the wakes. Invariants are also compared to the flux of kinetic energy and the production of turbulence kinetic energy, as these are quantities of importance in the wind turbine canopy layer. Rotational senses of the turbine rotors are varied in several configurations with effects in the flux of kinetic energy and the anisotropy of turbulence. Measurements of power and efficiency for each of the wind turbines are made following the procedure of Kang and Meneveau ${ }^{30}$ and Hamilton et al. ${ }^{31}$ The park efficiency is discussed in light of the anisotropy of turbulence encountered in the wind turbine canopy. It is expected that the performance of wind turbines arrays can be distinguished by the character of the turbulence in their inflows and wakes. The specific balance of stresses in the inflows to turbines correlates with the flux of kinetic energy, production of turbulence, and characteristic shapes of the anisotropy tensor.

\section{THEORY}

The Reynolds averaged Navier-Stokes equations for a wind turbine canopy or wake are simplified by omitting the unsteady term through ensemble averaging of the equations. All measurement locations considered in the experiment are sufficiently far from solid bodies that viscous terms in the local momentum balance can be neglected. The thrust force of the turbines in the flow must 
be included in the local balance and acts primarily in the streamwise direction. In all following equations, the coordinates are designated as $x$ (streamwise), $y$ (wall-normal), and $z$ (spanwise) directions. Capital letters indicate mean quantities and lower-case letters refer to zero-centered fluctuations about the mean. An overline is employed to indicate that an ensemble average of the product of two quantities has been taken. Multiplying the simplified Reynolds-averaged Navier-Stokes by the mean velocity yields the mean kinetic energy equation for the turbine canopy layer given as

$$
U_{j} \frac{\partial \frac{1}{2} U_{i}^{2}}{\partial x_{j}}=-\frac{1}{\rho} U_{i} \frac{\partial P}{\partial x_{i}}+\underbrace{\overline{u_{i} u_{j}} \frac{\partial U_{i}}{\partial x_{j}}}_{P_{i j}}-\underbrace{\frac{\partial \overline{u_{i} u_{j}} U_{i}}{\partial x_{j}}}_{F_{i j}}-\mathcal{F}_{x_{i}} .
$$

The left hand side of the Eq. (1) is comprised of the convective terms and is balanced on the right by the energy added to the flow through pressure gradients, the production of turbulence kinetic energy (TKE) $\left(P_{i j}\right)$, and the flux of kinetic energy $\left(F_{i j}\right)$ by turbulence, respectively. The last term of Eq. (1) is representative of energy removed from the flow by the action of a wind turbine rotor.

The balance of stresses within the turbulent flow field is of importance when considering terms from Eq. (1). The Reynolds stresses determine which components are responsible for production of turbulent kinetic energy and in which direction flux of kinetic energy occurs. In order to quantify preferential directions of the turbulent stress field, it is useful to define the Reynolds stress anisotropy tensor $a_{i j}$ according to the original development by Rotta, ${ }^{15}$

$$
a_{i j} \equiv \overline{u_{i} u_{j}}-\frac{2}{3} k \delta_{i j}
$$

where $\delta_{i j}$ is the Kronecker delta and $k=\frac{1}{2}\left(\overline{u^{2}}+\overline{v^{2}}+\overline{w^{2}}\right)$ is the turbulent kinetic energy. The anisotropy tensor is normalized by $2 k$ as

$$
b_{i j}=\frac{a_{i j}}{2 k}=\frac{\overline{u_{i} u_{j}}}{\overline{u_{k} u_{k}}}-\frac{1}{3} \delta_{i j} .
$$

The normalized anisotropy tensor $b_{i j}$ has by definition a zero trace as a consequence of its formulation. ${ }^{19,23}$ The traces of $b_{i j}^{2}$ and $b_{i j}^{3}$ are non-zero quantities and are related to the invariants $\eta$ and $\xi$ of the anisotropy tensor,

$$
\begin{aligned}
& 6 \eta^{2}=b_{i i}^{2}=b_{i j} b_{j i}, \\
& 6 \xi^{3}=b_{i i}^{3}=b_{i j} b_{j k} b_{k i} .
\end{aligned}
$$

An eigenvalue decomposition of $b_{i j}$ yields three eigenvalues whose sum is necessarily zero following the trace of the kernel $b_{i j}$, and a set of eigenvectors that correspond to an optimal basis describing the vector space of $b_{i j}$ given by,

$$
b_{i j}=\Sigma_{i j} \lambda_{i} \Sigma_{i j}^{-1}
$$

Eigendecomposition transforms the symmetric second-order tensor of the normalized Reynolds stress anisotropy into a set of principle components $\lambda_{i}$ and an associated vector basis $\Sigma_{i j}$. Because $b_{i j}$ is a symmetric tensor, the vectors in $\Sigma_{i j}$ are orthonormal and can be considered the optimal coordinate system $\left(x^{\prime}, y^{\prime}\right.$, and $\left.z^{\prime}\right)$ describing the vector space of $b_{i j}$.

$$
\Sigma_{i j}=\left\langle x^{\prime}, y^{\prime}, z^{\prime}\right\rangle .
$$

The invariants $\eta$ and $\xi$ can be expressed in terms of the eigenvalues directly as

$$
\begin{aligned}
\eta^{2} & =\frac{1}{3}\left(\lambda_{1}^{2}+\lambda_{1} \lambda_{2}+\lambda_{2}^{2}\right), \\
\xi^{3} & =-\frac{1}{2} \lambda_{1} \lambda_{2}\left(\lambda_{1}+\lambda_{2}\right) .
\end{aligned}
$$

Invariants of the normalized Reynolds stress anisotropy tensor are related to the degree of anisotropy $(\eta)$ and the characteristic shape described by the eigenvalues $(\xi) .{ }^{32}$ 


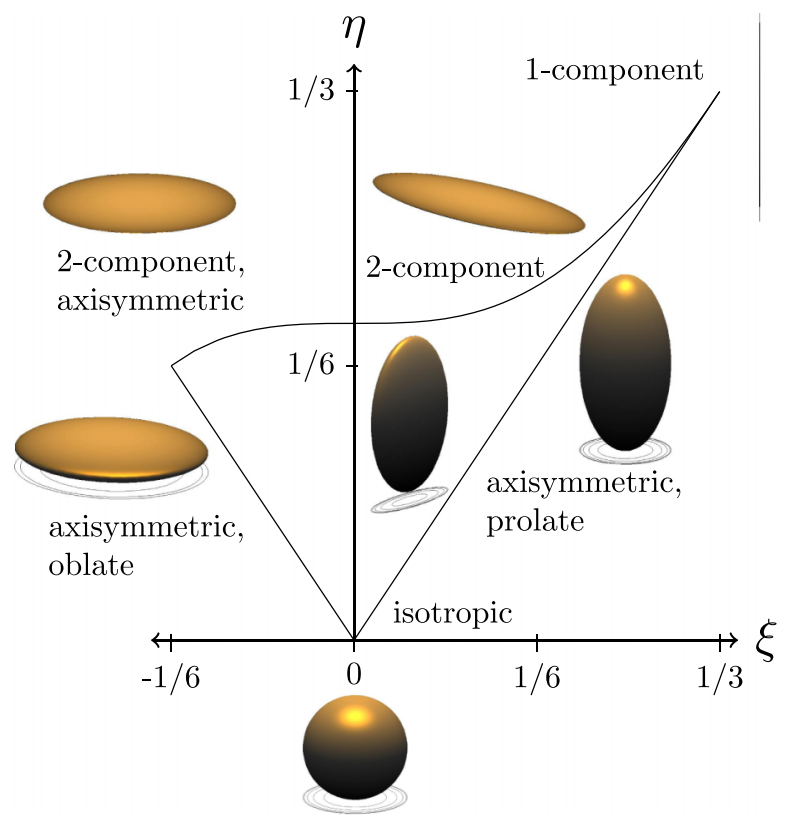

FIG. 1. Lumley triangle showing limits of invariants $\eta$ and $\xi$ and characteristic shapes of $b_{i j}$. Shapes for special cases correspond to those in Table I.

The invariants of $b_{i j}$ described by either Eqs. (4a) and (4b) or (7a) and (7b) can be shown graphically in the AIM. ${ }^{19}$ In Figure 1, theoretical limits are shown as vertices and edges of the triangle and represent special cases of turbulence.

The anisotropic state of turbulence can be described by a characteristic spheroid whose radii correspond to the eigenvalues $\lambda_{i}$ and is rotated with respect to the original Cartesian coordinate system to one described by the eigenvectors in Eq. (6). The shape of the characteristic spheroids correspond to the limits of "realizable" turbulence shown on the Lumley triangle in Figure 1 and described in the rightmost column of Table I.

The characteristic shapes associated with axisymmetric turbulence are either oblate or prolate spheroids for anisotropic turbulence. Oblate spheroids exhibit two eigenvalues that are of equal order of magnitude and one eigenvalue that is smaller resulting in a spheroid that is squeezed in one direction. Prolate spheroids show the opposite effect with one eigenvalue that is large compared to the other two, resulting in a spheroid that is stretched in one direction.

The special cases of turbulence outlined in Table I are often used in scaling and theoretical development but are not often observed in experiments. Nearly axisymmetric turbulence is common in wakes and is observed in the turbulence presented here. The shapes described in Table I are idealized, issuing from theoretical limits. The invariants of anisotropic turbulence seen in the experiment

TABLE I. Special states of turbulence given on the Lumley triangle. Limits of the Reynolds stress anisotropy tensor are shown in terms of matrix invariants and eigenvalues. Spheroidal radii correspond to principle components $\lambda_{i}$ from the eigenvalue decomposition of $b_{i j}$.

\begin{tabular}{lccl}
\hline \hline State of turbulence & Invariants & Eigenvalues of $b_{i j}$ & Shape of spheroid \\
\hline Isotropic & $\xi=\eta=0$ & $\lambda_{i}=0$ & Sphere \\
Two-component axisymmetric & $\xi=-\frac{1}{6}, \eta=\frac{1}{6}$ & $\lambda_{1}=\lambda_{2}=\frac{1}{6}$ & Disk \\
One-component & $\xi=\eta=\frac{1}{3}$ & $\lambda_{1}=\frac{2}{3}, \lambda_{2}=\lambda_{3}=-\frac{1}{3}$ & Line \\
Axisymmetric, one large eigenvalue & $\xi=\eta$ & $-\frac{1}{3} \leq \lambda_{1}=\lambda_{2} \leq 0$ & Prolate spheroid \\
Axisymmetric, one small eigenvalue & $-\xi=\eta$ & $0 \leq \lambda_{1}=\lambda_{2} \leq \frac{1}{6}$ & Oblate spheroid \\
Two-component & $\eta=\left(\frac{1}{27}+2 \xi^{3}\right)^{1 / 2}$ & $\lambda_{1}+\lambda_{2}=\frac{1}{3}$ & Ellipse \\
\hline \hline
\end{tabular}




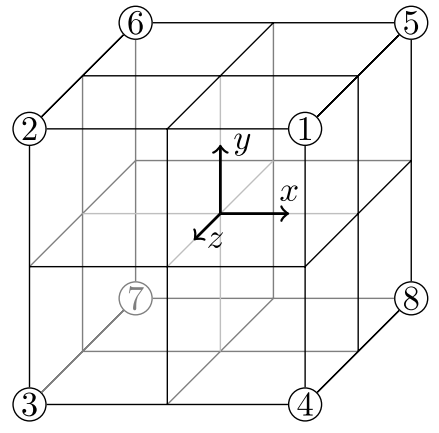

(a)

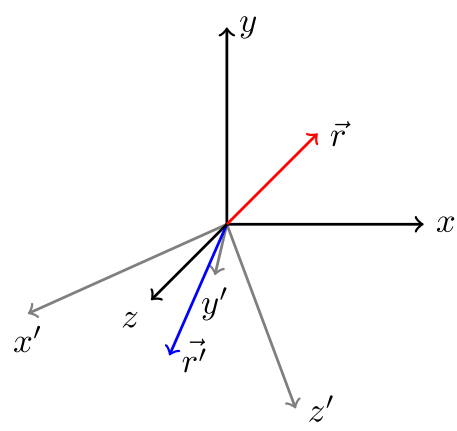

(b)

FIG. 2. Octants of the Cartesian coordinate system defined by the sign of standard vectorial components and rotated coordinate system and characteristic vectors of both the Cartesian system and the principle axes. (a) Octants defined in Cartesian coordinate system. (b) Characteristic vector, $\vec{r}$, for principle coordinate system.

fall within the central area of the Lumley triangle and the characteristic spheroids do not match exactly those in the table.

The principle axes output from the eigendecomposition in Eq. (5) can be concatenated to form a direct cosine matrix, denoted below as $R$. From the direct cosine matrix Euler angles $\left(\theta_{x}, \theta_{y}\right.$, and $\theta_{z}$ ) are extracted that map the initial Cartesian axes onto the set of principle axes for any given measurement location. The direct cosine matrix is expressed in terms of the Euler angles ${ }^{33}$ as

$R=\left[\begin{array}{ccc}\cos \theta_{y} \cos \theta_{z} & \cos \theta_{y} \sin \theta_{z} & \sin \theta_{y} \\ \cos \theta_{x} \sin \theta_{z}+\cos \theta_{z} \sin \theta_{x} \sin \theta_{y} & \cos \theta_{x} \cos \theta_{z}-\sin \theta_{x} \sin \theta_{y} \sin \theta_{z} & -\cos \theta_{y} \sin \theta_{x} \\ \sin \theta_{x} \sin \theta_{z}-\cos \theta_{x} \cos \theta_{z} \sin \theta_{y} & \cos \theta_{z} \sin \theta_{x}+\cos \theta_{x} \sin \theta_{y} \sin \theta_{z} & \cos \theta_{x} \cos \theta_{y}\end{array}\right]$.

Here, $\theta_{x}, \theta_{y}$, and $\theta_{z}$ represent rotations about the original $x, y$, and $z$ axes, respectively.

To determine where the principle axes reside with respect to the original coordinate system, a single vector $\vec{r}$ is defined central to the coordinate system such that its magnitude is unity and it is located equidistant from each of the unit vectors defining the coordinate axes.

$$
\vec{r}=\frac{1}{\sqrt{3}}\left\langle\vec{e}_{x}, \vec{e}_{y}, \vec{e}_{z}\right\rangle,
$$

where $\vec{e}_{i}$ are unit vectors in the $i$ direction

The vector $\vec{r}$ can be subjected to the three rotations from the direct cosine matrix. When $\vec{r}^{\prime}$ resides in a given octant, the principle coordinate system resides mainly in the same octant. The three dimensional Cartesian system is shown with conventionally defined octants in Figure 2(a). Rotating a set of unit vectors according to the Euler angles leads to the transformed system shown in Figure 2(b). The characteristic vector $\vec{r}$ is subjected to the same rotations and is likewise designated with a prime.

\section{EXPERIMENTAL SETUP}

The experiment was conducted in the closed-circuit wind tunnel at Portland State University (PSU). The test section of the wind tunnel measures $5 \mathrm{~m} \times 0.8 \mathrm{~m} \times 1.2 \mathrm{~m}$ in the $x, y$, and $z$ directions, respectively. Figure 3 shows the setup for the current experiment including several locations of velocity measurement.

The wind tunnel is furnished with a passive grid at the entrance of the test section consisting of 7 horizontal and 6 vertical rods. Vertical strakes were fabricated to extend the high-shear region of the inflow such that the velocity profile exhibits characteristics similar to the ABL. Surface roughness was added to the floor of the wind tunnel via chains with average diameter of approximately $0.0075 \mathrm{~m}$, spaced $0.11 \mathrm{~m}$ apart upstream of the turbine array. The wind turbine rotors have diameter $D=0.12 \mathrm{~m}$. The rotor diameter is used to normalize spatial coordinates in the analysis. Streamwise 


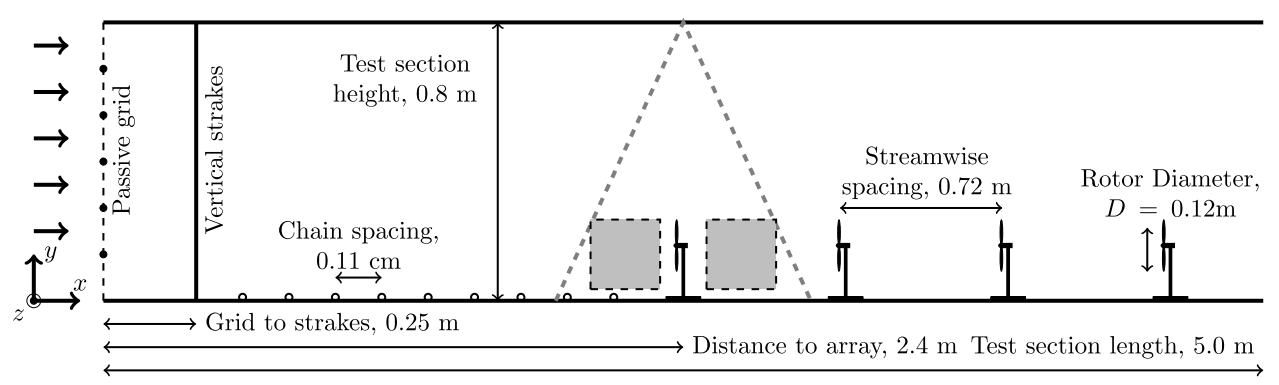

FIG. 3. Experimental setup shown in wind tunnel test section. Dimensions are for reference only; image not to scale.

gradients of mean velocities are orders of magnitude less than wall-normal gradients upstream of the entrance row turbine model indicating that the simulated boundary layer is well developed.

The turbulence intensities of the three components of velocity in the approach flow are highest near the wall, consistent with the turbulent boundary layer theory. The turbulence intensity is greatest in the streamwise direction, $\sigma_{u} /|U| \sim 0.2$. In the rotor area, the streamwise turbulence intensity is on the order of 0.15 , similar to that of previous wind tunnel experiments with turbine arrays. ${ }^{34,35}$ The vertical and spanwise components, $\sigma_{v} /|U|$ and $\sigma_{w} /|U|$, respectively, are smaller in magnitude than the streamwise component. The streamwise integral length scale is approximately $0.13 \mathrm{~m}$, which is the same order of magnitude as the turbine rotor and representative of conditions seen by full-scale turbines in atmospheric flows. For further detail on the inflow characterization, the reader is referred to Hamilton et al. . $^{35}$

Four configurations of a turbine array were tested in the wind tunnel with variations of the direction of rotation of the rotor blades. Figure 4 shows the arrangements undertaken in the current work. The configuration forming the basis of comparison is that of uniform rotation of turbine blades in Figure 4(a). Test cases were:

- Row-by-row alternation of rotational sense of the rotors, Figure 4(b).

- Column-by-column alternation, Figure 4(c). The center column of turbines in the array have clockwise $(\mathrm{CW})$ rotors, off-center columns have counter-clockwise $(\mathrm{CCW})$ rotors.

- Checkerboard case, Figure 4(d). Adjacent turbines have opposing rotational senses.

In all cases, the streamwise spacing of turbine rows was maintained as a constant $S_{x}=6 * D$ and the spanwise turbine spacing within the rows was fixed at $S_{z}=3 * D$.

During the course of the experiment, the wind tunnel speed was maintained constant where the hub height velocity was $U_{\text {hub }}=4.75 \mathrm{~m} / \mathrm{s}$. The Reynolds number based on this velocity and the diameter of the wind turbine rotor was $\operatorname{Re}_{D}=U_{\text {hub }} D / v=3.8 \times 10^{4}$. A Reynolds number at this

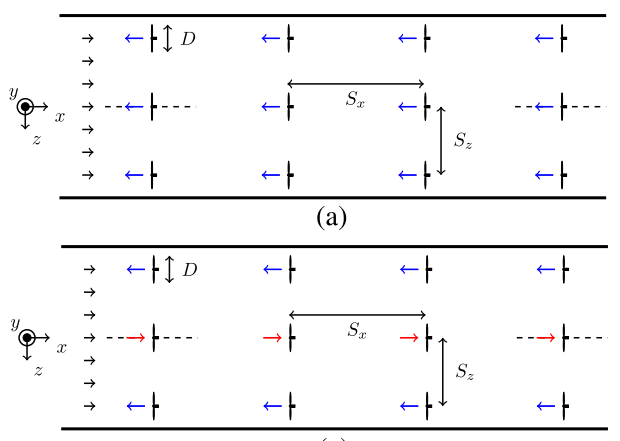

(c)

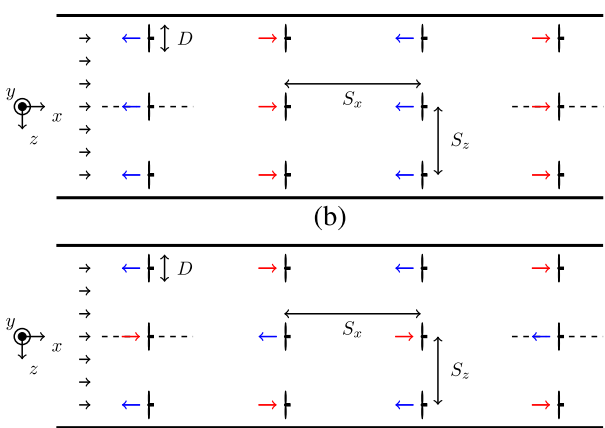

(d)

FIG. 4. Configurations of the wind turbine array tested in the PSU wind tunnel. The colored rotation vector indicates rotational sense of the turbine rotors according to the right-hand-rule. Red vectors indicate clockwise rotation; blue vectors indicate counter-clockwise rotation. Dashed lines indicate SPIV measurement planes. (a) Uniform rotation. (b) Row-by-row arrangement. (c) Column-by-column arrangement. (d) Checkerboard arrangement. 


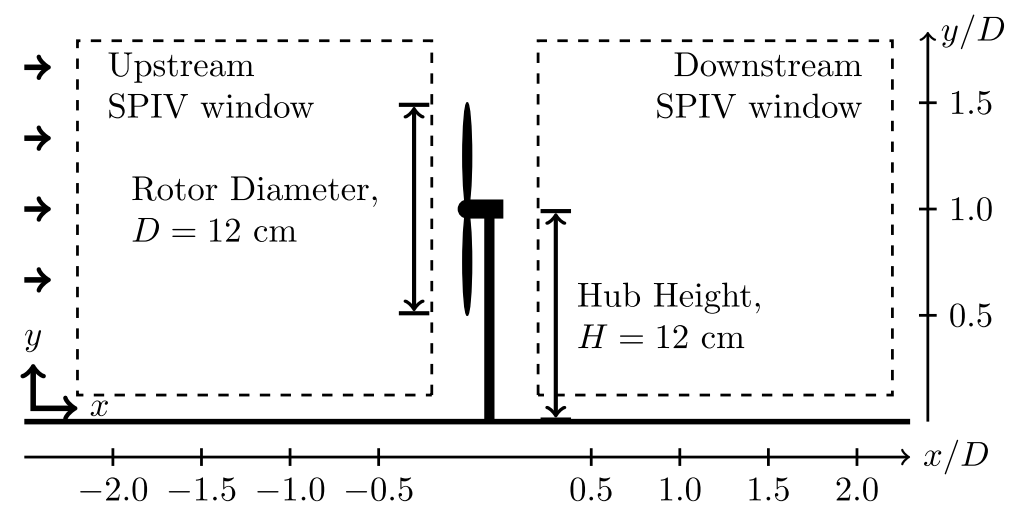

FIG. 5. Detail view of SPIV measurement areas located directly upstream and downstream of wind turbine models.

level is close to the range where main flow statistics in the wakes of wind turbines become Reynolds number independent as suggested by Chamorro and Arndt. ${ }^{36}$

Flow field measurements were made with two-dimensional/three-component (2D-3C) stereo particle image velocimetry (SPIV). The SPIV data in this experiment were collected in two windows simultaneously (Figure 5) directly upstream and downstream of the entrance and exit row turbines in the arrays, shown as dashed lines in Figure 4. A LaVision flow measurement system consisting of an Nd:Yag (532 nm, $200 \mathrm{~mJ}, 4$ ns duration) pulsed laser and with four CCD cameras (4 MP resolution) arranged in pairs for the two SPIV interrogation areas. The flow was seeded with neutrally buoyant droplets of diethylhexyl sebacate and allowed to mix thoroughly. Seeding was kept at a constant level throughout data collection to ensure resolution of data and mitigate inconsistency in vector calculation. The laser sheet was approximately $0.002 \mathrm{~m}$ thick with a transverse divergence angle of less than $5 \mathrm{mrad}$ across the span of the areas of interest. Measurement locations are shown in Figure 3 and a detailed view is seen in Figure 5. A single laser sheet was used for both measurement locations to ensure alignment of the fields upstream and downstream of each turbine.

Raw images were processed into vector fields using a multi-pass FFT-based correlation algorithm. The interrogation window reduced in size with two passes each at $64 \times 64$ and $32 \times 32$ pixels with a 50\% overlap. The final pass resulted in a spatial resolution of approximately $1.5 \mathrm{~mm}$ between vectors. The time delay between laser pulses and respective image acquisitions was set to $250 \mu$ s to achieve an average particles shift of 8 pixels between successive frames. For each measurement location, a total of 2000 SPIV snapshots were recorded to ensure convergence of statistics.

The wind turbine models consisted of a steel mounting plate $0.075 \mathrm{~m}$ thick, a hollow steel mast, and rotor blades cut from $0.0005 \mathrm{~m}$ sheet steel. The blades were pitched approximately $\gamma_{\text {root }}=20^{\circ}$ out of the rotor plane at the root of the blade and had a $5^{\circ}$ twist from root to tip, resulting in a pitch of $\gamma_{\text {tip }}=15^{\circ}$ at the tip of each blade. The nacelle of each turbine was comprised of a DC electric motor (Faulhaber GMBH \& Co., Series 1331T012SR). Figure 6 shows a schematic of the fully assembled turbine models including mast, nacelle, rotor, and mounting plate.

Measurements of power and efficiency were made by way of a torque sensor fixed to the wind turbines. The reader is referred to Ref. 31 for a detailed discussion of the power measurements. During the collection of flow field measurements, the torque sensor was not in place to remove effects added to the flow.

\section{RESULTS}

The discussion of mean statistics and invariants of the normalized Reynolds stress anisotropy tensor below is undertaken in several steps. To establish the differences of individual turbines with either $\mathrm{CW}$ or $\mathrm{CCW}$ rotors, results are shown for two entrance row turbines. The entrance row observed the atmospheric-like boundary layer flow. These results are contrasted with turbines in 


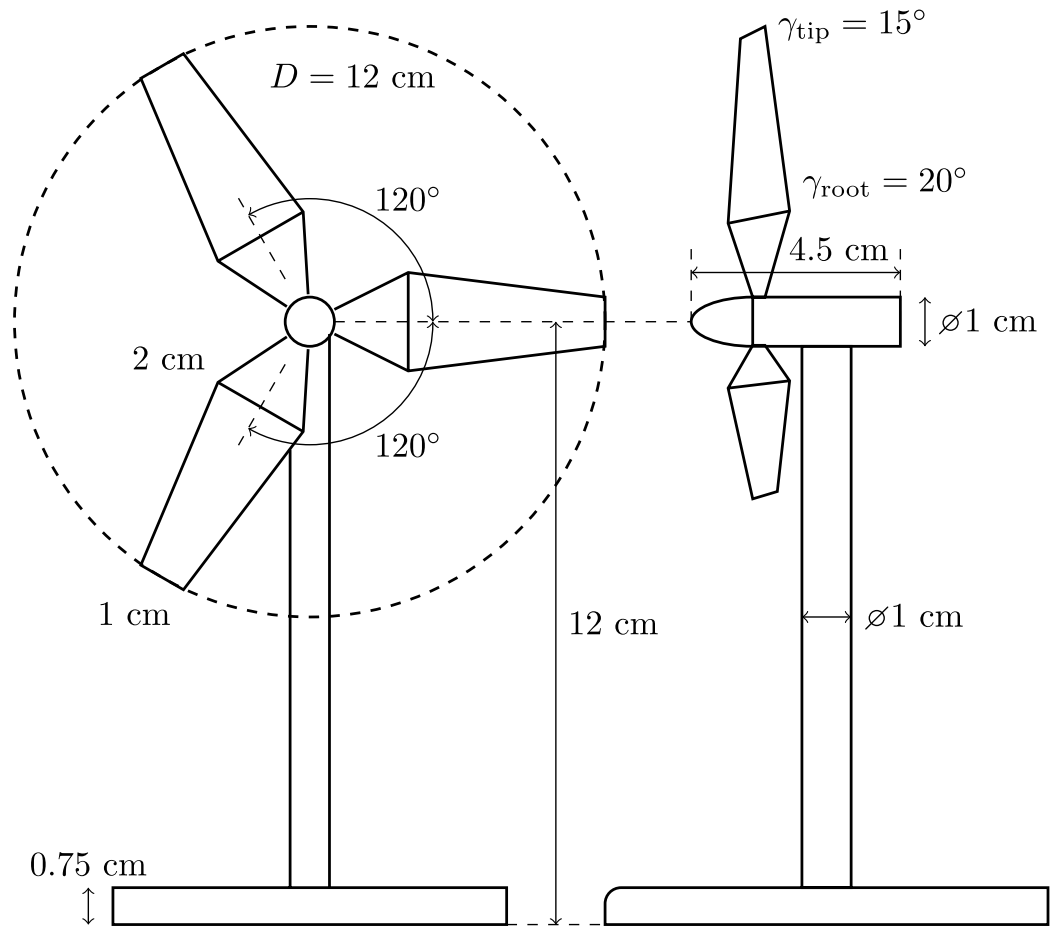

FIG. 6. Technical drawing of model wind turbines used in experiments. The mounting plate spans the full width of the wind tunnel although only a small section is shown.

the fully developed region of the array, taken to be the exit row turbines. There, the measurements necessarily include the cumulative effects of the array configurations.

\section{A. Single wind turbines}

Results for entrance row turbines are shown in Figure 8. The layout of contour plots in each subfigure is depicted in Figure 7 including rotational sense of the rotor for reference. In each of the subfigures, the top row of contour plots shows the statistics of the entrance row of the uniform case (CCW rotor, top row) and the checkerboard arrangement (CW rotor, bottom row). Measurements around the entrance rows of the row-by-row arrangement and the column-by-column arrangement are statistically identical to the others and have been omitted from the discussion of results for brevity. Differences seen in the wakes arise from measurement error (estimated to be less than 3\% in

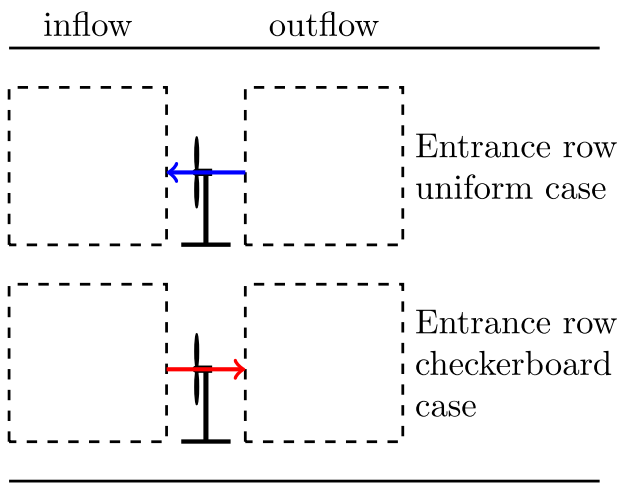

FIG. 7. Rotational sense and arrangement of results for turbines in subfigures $8(a)-8(h)$. 

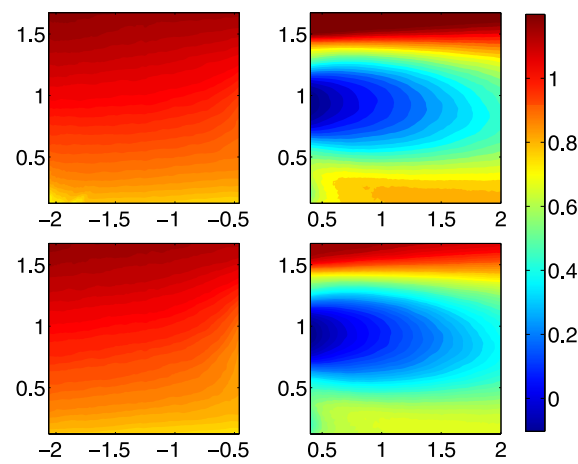

(a)
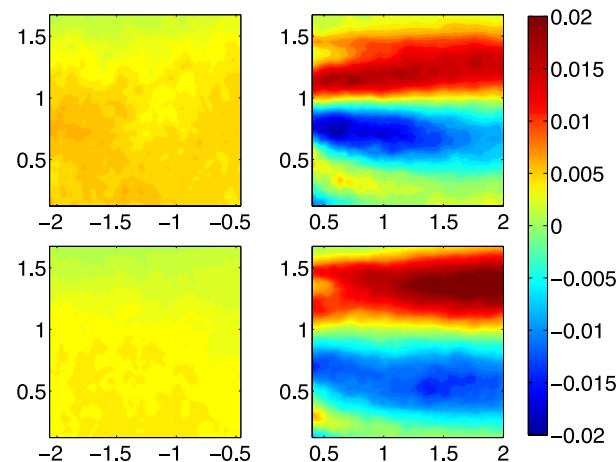

(c)
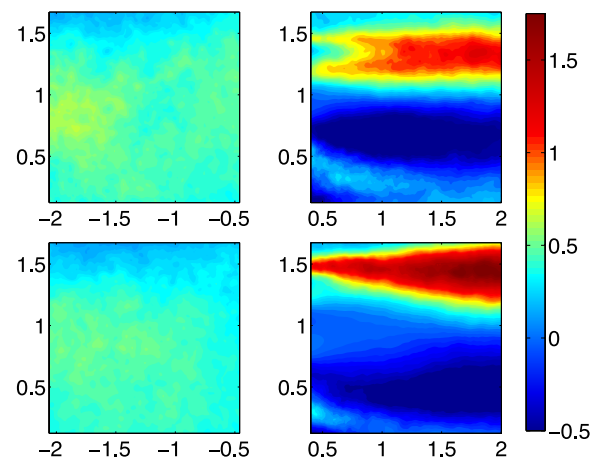

(e)
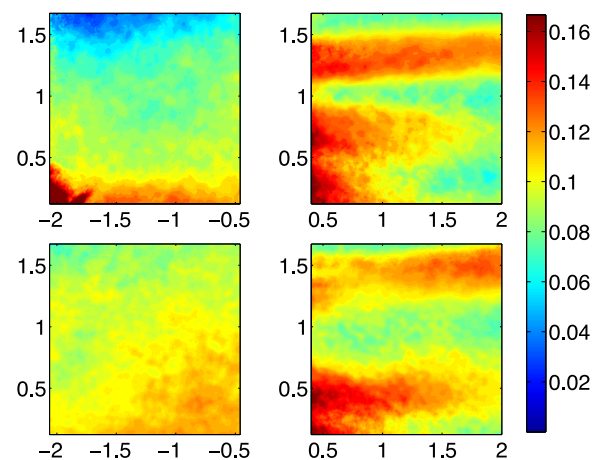

(g)
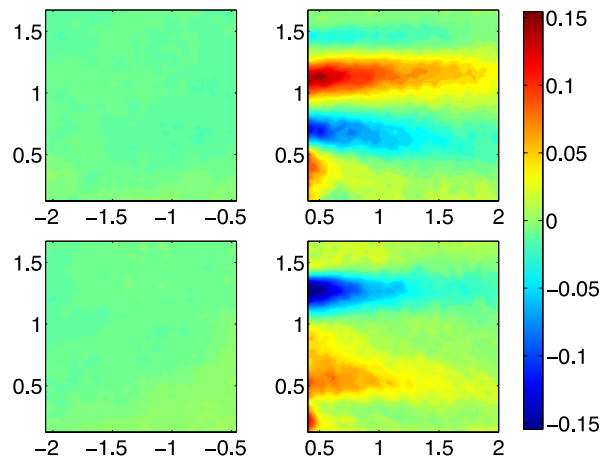

(b)
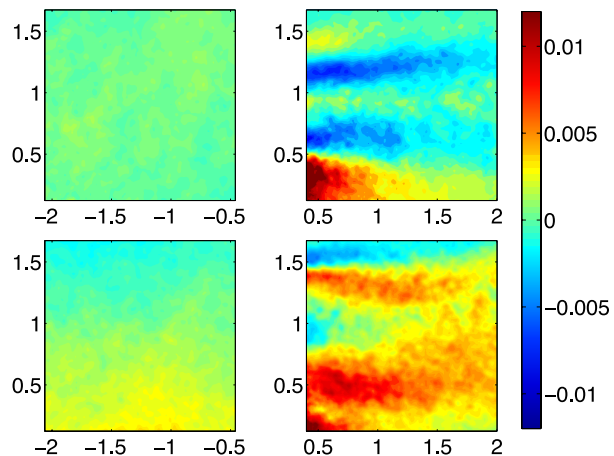

(d)
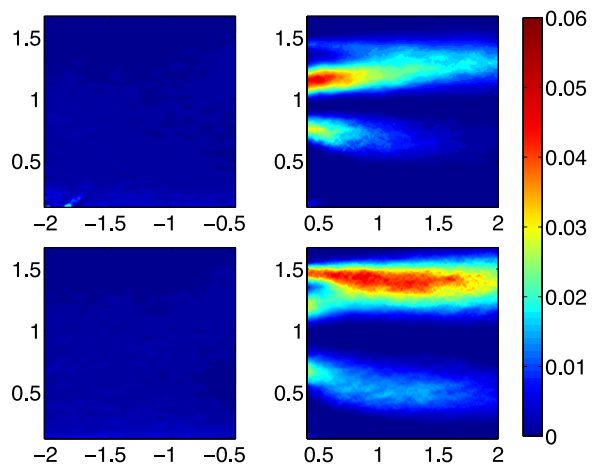

(f)
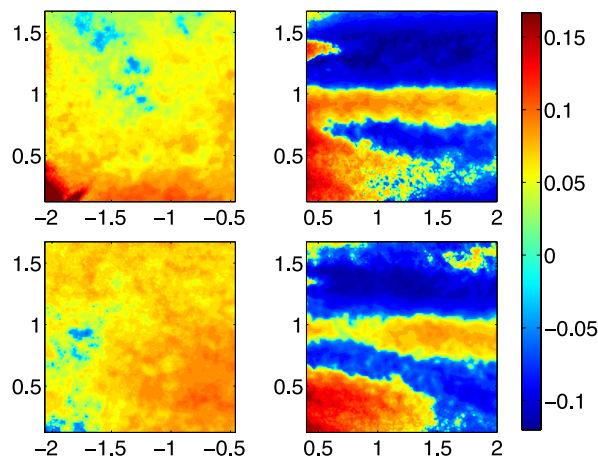

(h)

FIG. 8. Contours of data for entrance row turbines. In all subfigures, inflow and wake are shown for the CCW rotor (uniform arrangement) in the top row and for the $\mathrm{CW}$ rotor (checkerboard arrangement) in the bottom row. Entrance row turbines are

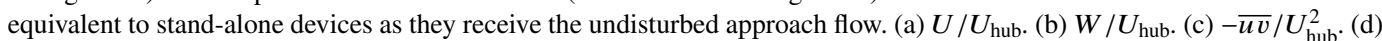
$-\overline{v w} / U_{\mathrm{hub}}^{2}$. (e) $F_{12}$. (f) $P_{12}$. (g) $\eta$. (h) $\xi$. 
the current experiment) and from the sensitivity of second-order statistics and numerical derivatives to small discrepancies in data sets.

Mean velocities and Reynolds stresses are normalized by the hub-height velocity of the approach flow $U_{\text {hub }}=4.75 \mathrm{~m} / \mathrm{s}$. The mean streamwise velocity $U / U_{\text {hub }}$ for entrance row wind turbines is shown in Figure 8(a). The left column of contour plots corresponds to the approach flow to the arrays and match upstream $(x / D \leq-1.5)$ of the model array. The wakes of the entrance row turbines demonstrate slight differences between array configurations. The wake of the CW rotating turbine in the checkerboard case shows a velocity deficit approximately $8.6 \%$ greater than the $\mathrm{CCW}$ rotor found in the uniform case.

The mean wall-normal velocity, $V / U_{\text {hub }}$ (not shown), features consistent trends regardless rotational sense of the turbine rotor. The vertical velocity in inflow measurement windows reflects the blockage seen in $U / U_{\text {hub }}$ as flow around the turbine rotor, upward above the nacelle and downward below. In each of the wakes, there is a mean vertical velocity into the center of the wake.

Spanwise velocity $W / U_{\text {hub }}$ shows the rotational sense of the turbine rotors, depicted in Figure 10(b). The turbine with CCW rotors (uniform case) shows a positive spanwise velocity (toward the reader) associated with the top-tip of the rotor and a negative spanwise velocity associated with the bottom-tip of the rotor. The opposite is seen for the CW rotor. Another distinction between the $\mathrm{CW}$ and $\mathrm{CCW}$ turbine rotors in the entrance row is that the magnitude of the spanwise velocity is moderately greater in the uniform case than the checkerboard case, opposite from trends of the streamwise velocity. The discrepancies between flow fields associated with $\mathrm{CW}$ and CCW rotors arise from slight differences in the geometry of the rotors produced in manufacturing.

Two of the Reynolds stresses normalized by the square of the hub-height velocity in the approach flow are shown in Figures 8(c) and 8(d). The in-plane Reynolds stress $-\overline{u v} / U_{\text {hub }}^{2}$ shows expected trends in each of the wakes where positive values are seen associated with the wake above hub-height and negative below. These results are quite similar to previous experiments with wind turbine arrays under similar conditions. . $^{2,31,34-36}$

The Reynolds stress composed of the ensemble average of fluctuations in the vertical and spanwise directions (Figure 8(d)) shows dependence on rotational sense of the rotors. In the wake area, rotors with a CCW orientation demonstrate negative values associated with the top- and bottom-tips of the rotor. The opposite is seen for turbines with a $\mathrm{CW}$ rotational sense, although magnitudes are nearly equal. Spanwise fluctuations have the same algebraic sign of those in the vertical velocity for $\mathrm{CCW}$ blade rotation, resulting in negative values of $-\overline{v w} / U_{\text {hub }}^{2}$ in the wake. For the $\mathrm{CW}$ rotor, the fluctuations have the opposite algebraic signs, resulting in positive values of $-\overline{v w} / U_{\text {hub }}^{2}$

The flux of mean kinetic energy by turbulence into the wakes of wind turbines is an indication of the entrainment of high-momentum flow into the turbine rotor area of the canopy. This has been shown in other wind turbine studies ${ }^{1,2,31}$ to be one of the primary mechanisms responsible for resupply of kinetic energy to the fully developed or infinite wind farm. The in-plane component of the flux tensor $F_{12}=-\overline{u v} U$ is the primary contributor to the overall flux and is seen in Figure 8(e). Other components of the flux tensor are one to two orders of magnitude smaller than $F_{12}$ and therefore not discussed in the current work. The flux in the wake of the CW rotor (Figure 8(e) bottom right) $F_{12}$ shows values that are approximately $10 \%$ larger than for the $\mathrm{CCW}$ rotor. Differences are attributed to discrepancies in the mean streamwise velocity and the in-plane Reynolds stress.

The production of TKE $P_{i j}$ makes a smaller contribution to the overall energy balance in Eq. (1) than the flux of kinetic energy. As with the flux tensor, only the in-plane component of production $P_{12}=-\overline{u v} \partial U / \partial y$ is included here as it represents the greatest contribution to the tensor, approximately $65 \%$ of the production described by the components available through the current measurements. The formulation of $P_{12}$ requires that both the in-plane Reynolds stress and a wall-normal gradient of the mean streamwise velocity be present to generate TKE. This is shown in the contour plots of Figure 8(f). Following the trends of $-\overline{u v} / U_{\text {hub }}^{2}$ and $F_{12}$, the greatest value of production occurs following the top-tip of the rotor for the checkerboard case and the root of the top rotor blade in the uniform case. There is a lesser contribution to the production of TKE following the lower half of the rotor areas and for $x / D \geq 1.5$. There the contributions are lower due to the softened gradients of the streamwise velocity. 
Non-zero turbulent shear stresses suggest that the stress tensor is not in its principle form and that the standard definition of $k$ may not communicate the full energy of turbulence present within a flow. Further, it indicates that there may be a more efficient coordinate system for describing the space of turbulent stresses. The normalized Reynolds stress anisotropy tensor $b_{i j}$ is decomposed in order to obtain eigenvalues and invariants $\eta$ and $\xi$ in the measurement fields according to Eq. (7).

Invariants of $b_{i j}$ are shown in Figures $8(\mathrm{~g})$ and $8(\mathrm{~h})$. In the approach flows, the second invariant $\eta$ generally decreases with wall-normal coordinate $y / D$, agreeing with the analysis of the anisotropy tensor presented by Smalley et al. ${ }^{21}$ and Leonardi et al. ${ }^{22}$ The region of very high $\eta$ in the bottom left corner of the measurement window showing the approach flow to the uniform case arises from artifacts in the SPIV measurements. The numerical sensitivity of the eigenvalue decomposition of $b_{i j}$ amplifies measurement error associated with these artifacts.

Theory of continuum mechanics ${ }^{32}$ indicates that the second invariant of a stress tensor $\eta$ is related to the degree of anisotropy of the tensor. Several conclusions can then be drawn regarding the contour plots of $\eta$ seen in Figure $8(\mathrm{~g})$. The most prevalent trend seen in the figure is a region of large $\eta$ trailing the top-tip of the rotor in each case. This indicates that the turbulence above $y / D \approx 1.25$ of the turbine rotor canopy is the most anisotropic seen in the wake. The minimum values of $\eta$ occur following the nacelle and above the wake. This is also the region where shear stresses are at a minimum for each of the wakes, implying that the turbulence stress tensor may be closer to its principle form prior to decomposition.

The third invariant of $b_{i j}$ is shown in Figure 8(h). The wake areas evidence positive values of $\xi$ following the nacelle and negative regions following the tips of the rotors. From the Lumley triangle in Figure 1, positive values of $\xi$ lead to a prolate spheroid and negative values lead to an oblate shape. For entrance row wind turbines, the characteristic spheroids tend toward prolate shapes following the nacelles and masts, regardless of rotational sense of the rotors. Following the area of the rotors above the hubs, spheroids are overwhelmingly oblate. Comparing these plots to those of $F_{12}$ indicates that the flux into the wakes (from either above or below) is associated with turbulence exhibiting two eigenvalues of similar magnitudes and one that is smaller. The connection between quantities from Eq. (1) and the invariants of $b_{i j}$ is discussed further in Sec. IV D.

\section{B. Wake statistics for array configurations}

Results discussed in the remainder of the article focus on the behavior the fully developed wind turbine array. The presentation of results follows the same order as for the individual turbines discussed above. However, because the inflows to exit row turbines must include cumulative effects of the array configurations, all four arrangements are significant for discussion. Contours plots of each quantity are shown in Figures 10 and 11 following the layout in schematic in Figure 9, which includes the rotational sense of exit row turbines for reference.

Inflows to exit row turbines show velocity deficits on the order of $30 \%$ as compared to the approach flow to the model turbine array (Figure 10). These windows are also representative of the far wakes of the third row of turbines and show $0.6 \leq U / U_{\text {hub }} \leq 0.8$. The flow blockage of the exit row of turbines is seen from $-0.75<x / D<0$. The inflows for the uniform and checkerboard cases show magnitudes in streamwise velocity approximately $7 \%$ larger than the row-by-row and column-by-column cases.

Exit row turbines show similar orders of magnitude of $W / U_{\text {hub }}$ at both top- and bottom-tip heights regardless of direction of rotation shown in figure. The mean spanwise velocity in the area directly behind the masts of the turbine models is negative, opposite of the entrance rows. The regions of distinct spanwise velocity also extend farther into the wake suggesting that turbines in the fully developed region of the array impart a greater mean out-of-plane motion than in leading rows.

The normalized in-plane shear stress $-\overline{u v} / U_{\text {hub }}^{2}$ in Figure 10 (c) shows consistent trends for each of the wakes of exit row turbines. The inflow to fourth row turbines is very similar in all cases $\left(-2 \times 10^{-3} \leq-\overline{u v} / U_{\text {hub }}^{2} \leq 7 \times 10^{-3}\right)$, regardless of rotational sense of upstream turbines. As the in-plane Reynolds stress does not demonstrate dependence on rotational sense of the rotors, it is expected that it is consistent in each case. In the wakes of the exit row, there is an increase in magnitude $(\sim 15 \%)$ for turbines rotating in the $\mathrm{CW}$ sense. These differences are evidenced in the 


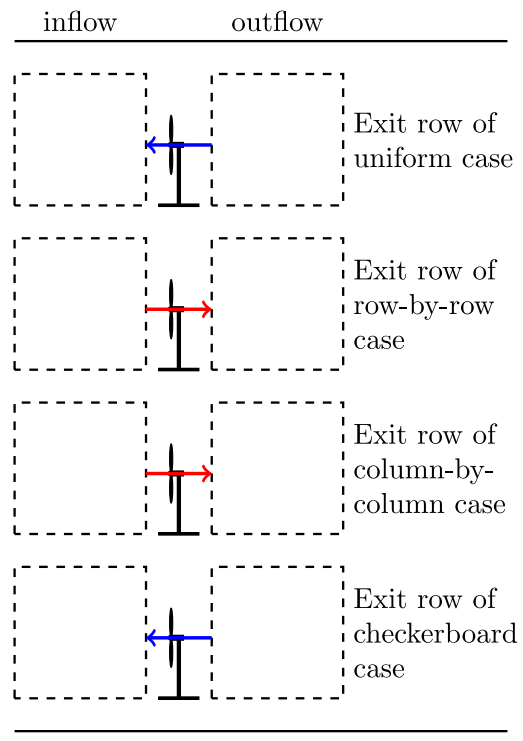

FIG. 9. Rotational sense of exit row turbine rotors and order of results for Figures 10 and 11.

region of the wake following the top-tip of the rotor. Magnitudes of the shear stresses correlate well with gradients in the mean flow between regions of the wakes and the canopy as shown in Figure 10(a).

Rotational sense stands out in the shear stress $-\overline{v w} / U_{\text {hub }}^{2}$, consistent with the entrance row turbines. Figure 10 (d) demonstrates that $-\overline{v w} / U_{\text {hub }}^{2}$ is quite uniform and rather small in the inflows to exit row of turbines. This shear stress component is short-lived compared to the in-plane shear stress. When the rotor blade is moving in the CCW sense, the regions of high stress associated with the top- and bottom-tips of the rotor extend farther in the streamwise direction as compared to those of $\mathrm{CW}$ rotors.

The in-plane Reynolds stress remaining in the far wake of third row turbines (inflows in Figure 10(c)) leads to a continued and significant contribution to the overall energy balance by the flux term. This is especially evident for $y / D \geq 1$ in the inflows to exit row turbines. The presence of positive flux indicates that although inflows show a momentum deficit compared to the entrance row, they also exhibit entrainment of high-momentum flow into the swept area of the rotor. As discussed earlier, a positive value of the in-plane flux implies entrainment of high-momentum flow downward from above. Conversely, a negative value of flux indicates entrainment from below the rotor area upward.

In the wakes of turbines in the fully developed region, $F_{12}$ is similar in form to the entrance row, although the magnitudes are somewhat reduced. The $\mathrm{CW}$ rotors demonstrate slightly increased turbulence in the wakes and thus slightly greater values of flux. The maximum values of $F_{12}$ are approximately $1.3 \mathrm{~m}^{3} / \mathrm{s}^{3}$, occurring at $x / D \approx 1.5$ into the wake. The flow below the rotors is constrained by the floor of the wind tunnel and experiences the development of a boundary layer between each row of wind turbines. The reduced mean velocity near the wall limits the momentum available for upward flux into the wake. For the arrays discussed in the current work, upward flux is approximately $1 / 2$ of that observed in the upper canopy.

Inflows to the exit row show $P_{12}$ to be nearly null and confirm that the production of TKE is isolated to the near wake. $P_{12}$ following the rotor at bottom-tip height is approximately $0.015 \mathrm{~m}^{2} / \mathrm{s}^{3}$ for all cases. The wake of the row-by-row configuration shows peak values of $P_{12}$ on the order of $0.05 \mathrm{~m}^{2} / \mathrm{s}^{3}$ approximately twice that of the uniform and checkerboard cases. Peak values occur within one rotor diameter at top-tip height in all cases. The difference in peak values of $P_{12}$ is attributed to a variation in $-\overline{u v}$ between cases. The gradient $\partial U / \partial y$ changes less than $10 \%$ between array configurations and cannot account for the difference in production by itself. $P_{12}$ shows extreme values in the wakes of exit row turbines to be approximately $85 \%$ of those in the entrance 

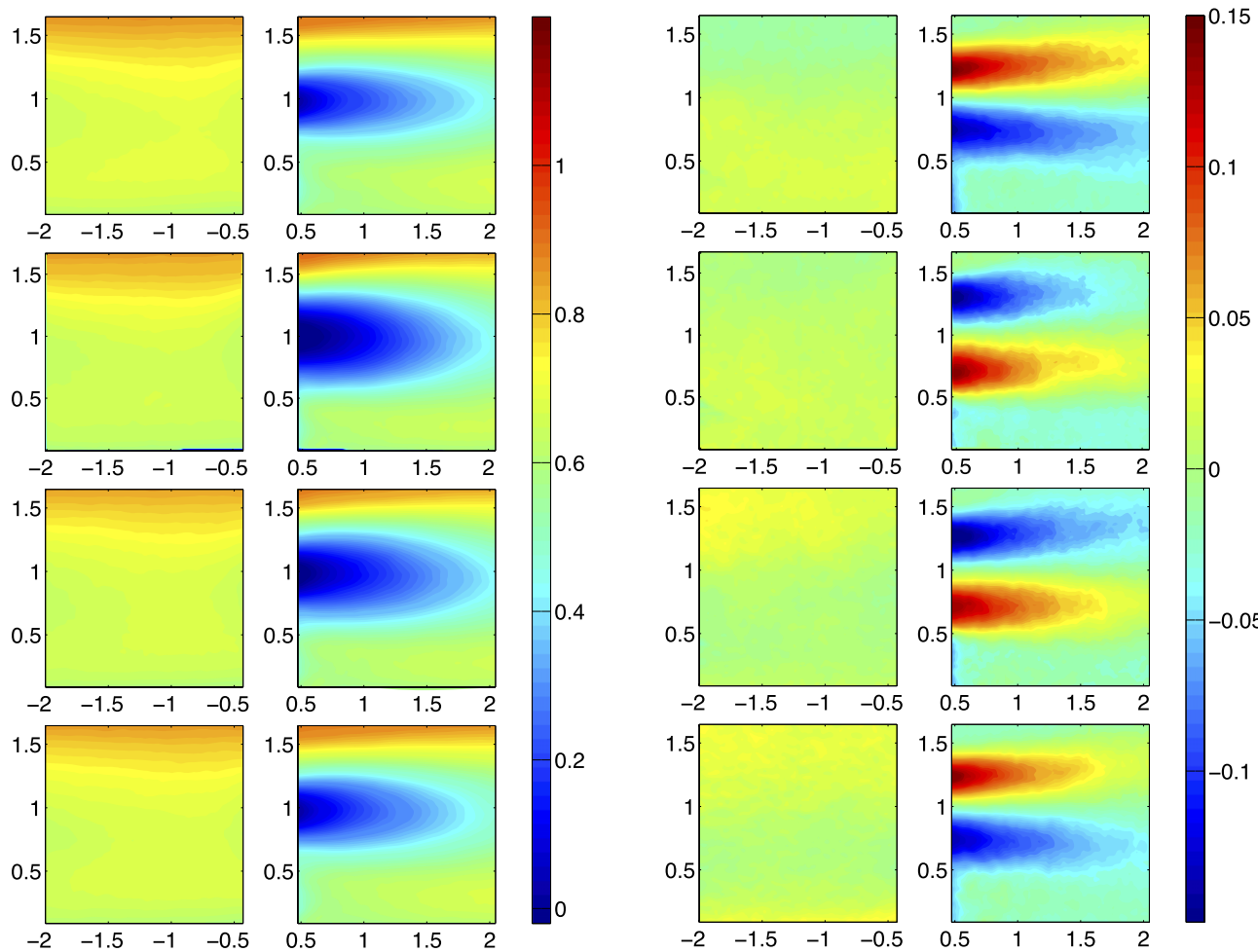

(a)
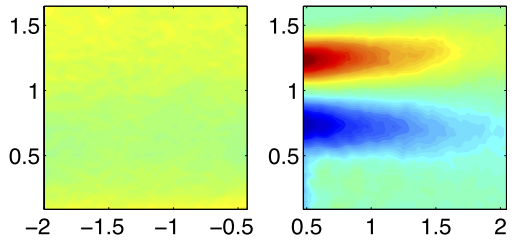

(b)
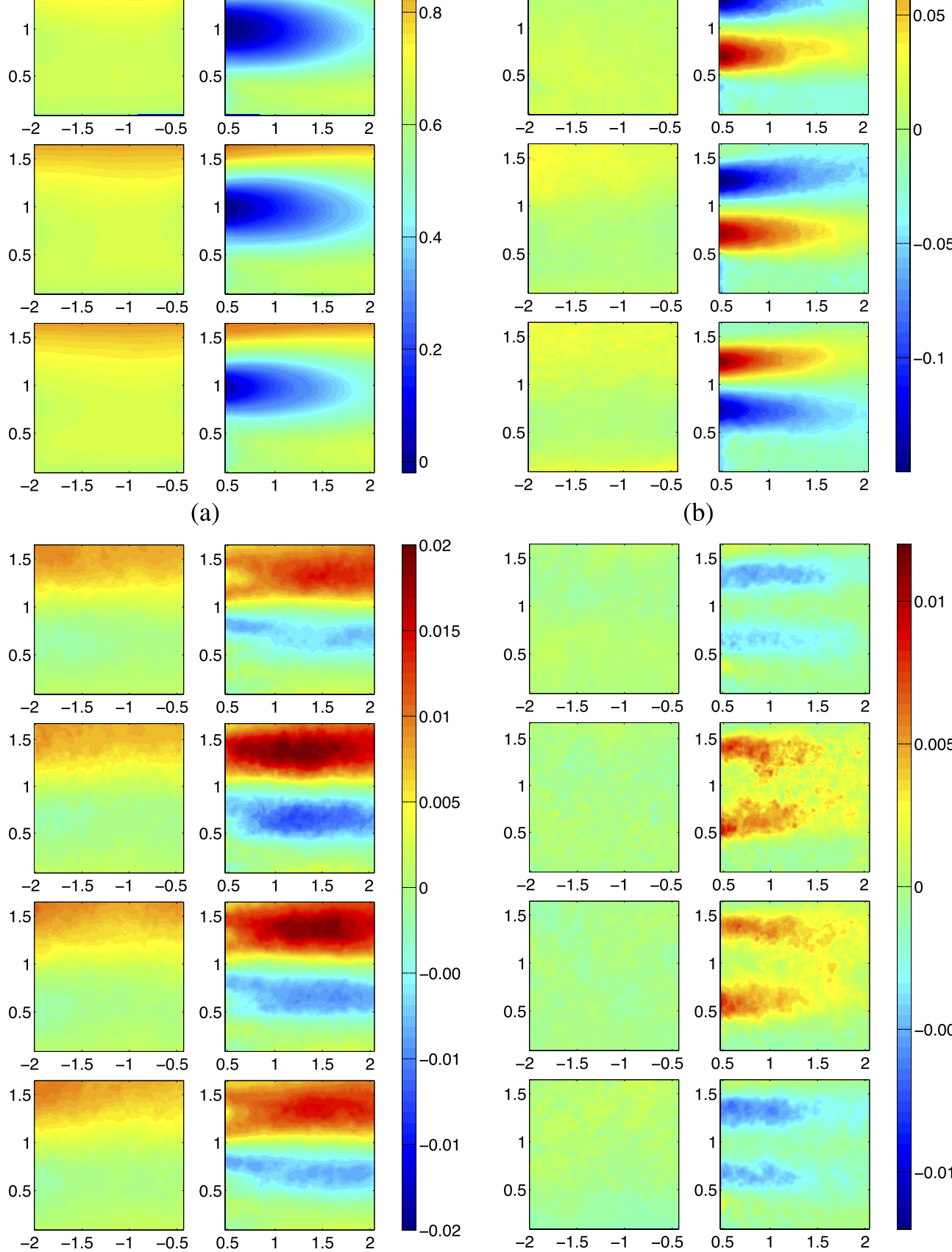

(c)
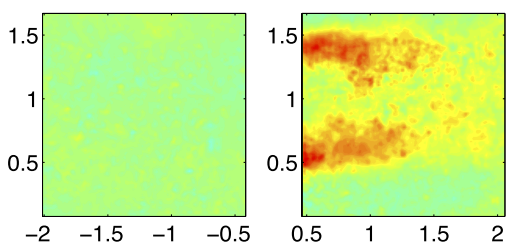

$-0.005$
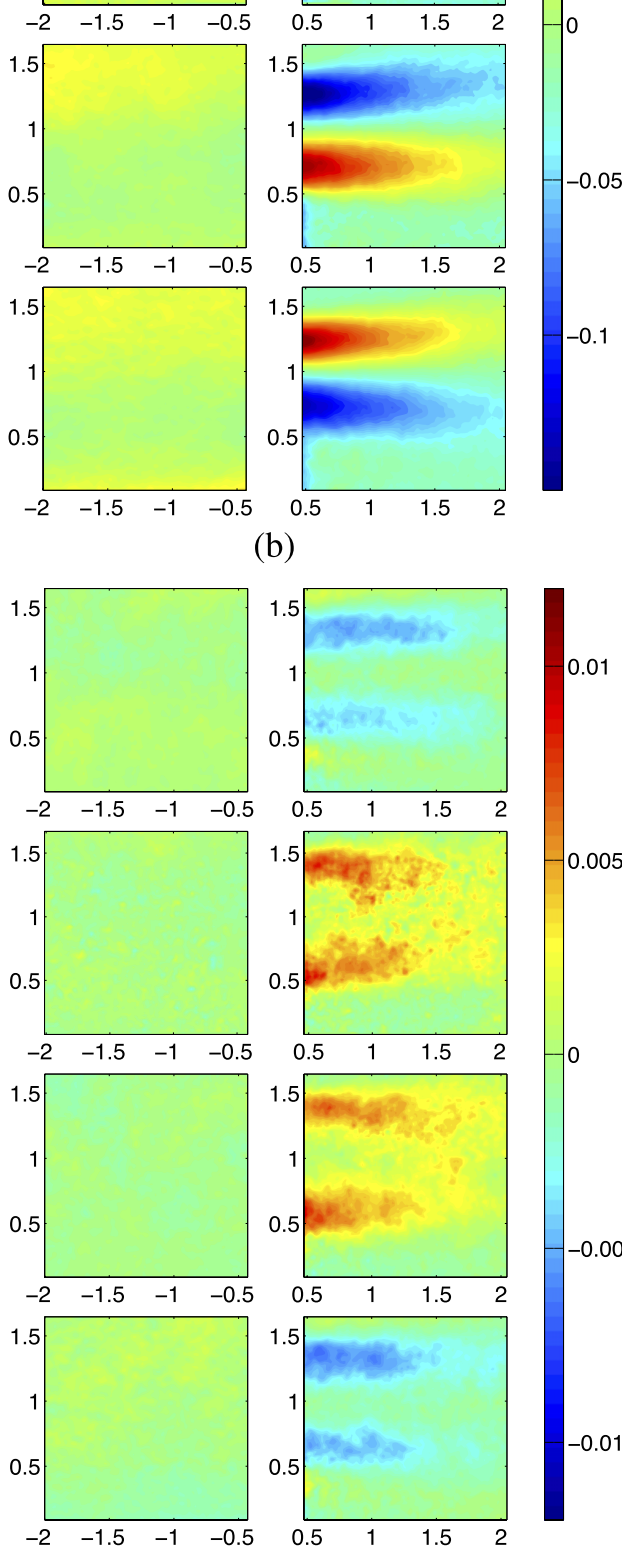

(d)

FIG. 10. Contour plots of mean velocities and Reynolds stresses for exit row turbines in each array configuration. (a) Mean

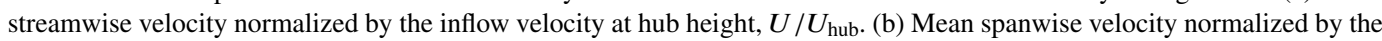
inflow velocity at hub height, $W / U_{\text {hub. }}$ (c) Mean in-plane Reynolds stress $-\overline{u v} / U_{\text {hub }}^{2}$. (d) Mean vertical-spanwise Reynolds stress $-\overline{v w} / U_{\text {hub }}^{2}$. 

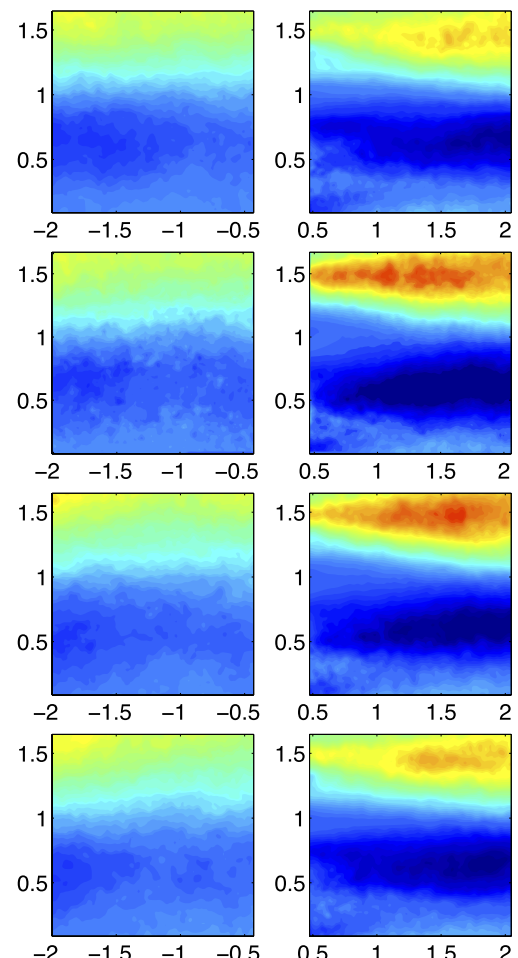

(a)
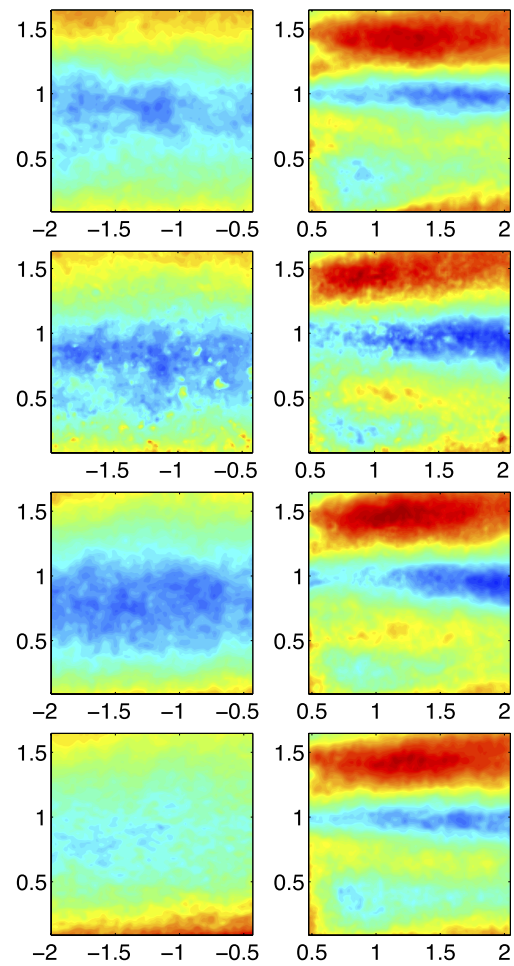

(c)
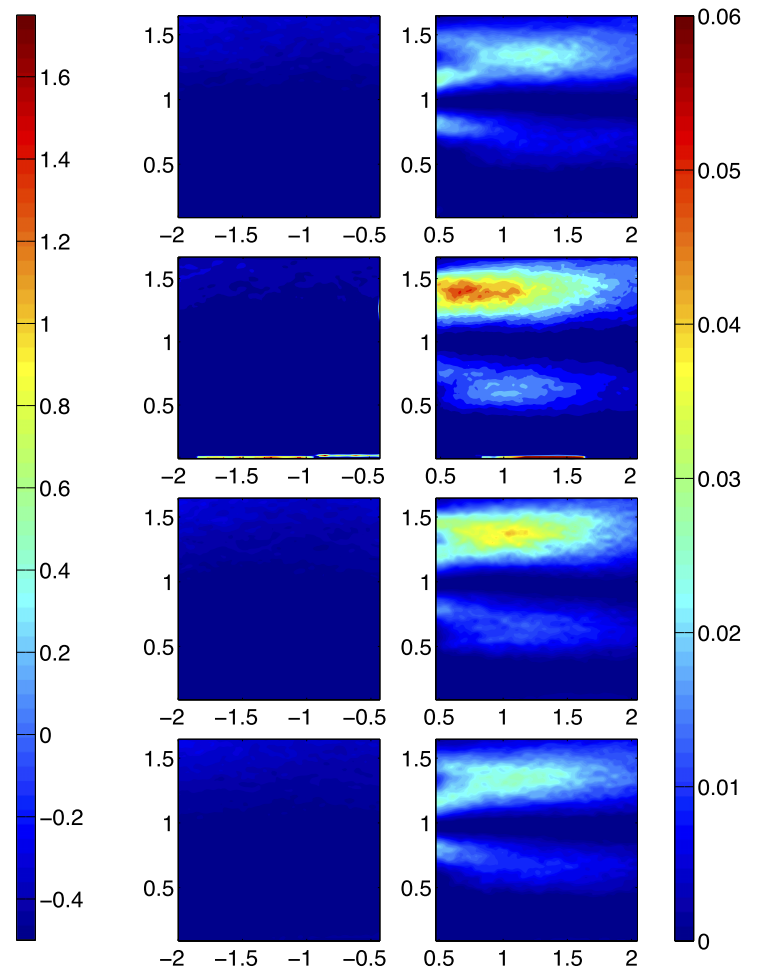

(b)
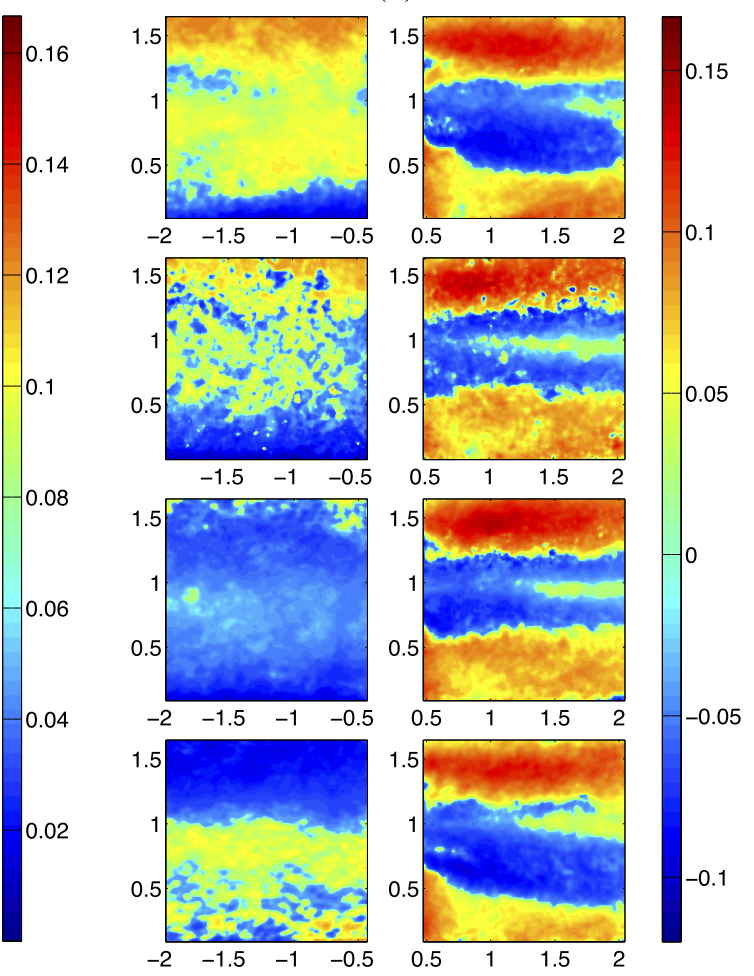

(d)

FIG. 11. Contour plots of terms from Eq. (1), flux of kinetic energy 11(a) and production of TKE 11(b), and invariants of the normalized Reynolds stress tensor, $\eta 11$ (c) and $\xi 11$ (d). (a) Mean in-plane flux of kinetic energy, $F_{12}=-\overline{u v} U$. (b) Mean in-pane production of TKE, $P_{12}=-\overline{u v} \frac{\partial U}{\partial y}$. (c) Plots of second invariant of the normalized anisotropy tensor, $\eta$. Corresponds to the ordinate of the Lumley triangle. (d) Plots of third invariant of the normalized anisotropy tensor, $\xi$. Corresponds to the abscissa of the Lumley triangle. 
row. Interesting to note is that the inflows are nearly zero for both entrance and exit rows. In the approach flow, the gradient $\partial U / \partial y$ is visible in the ABL but the in-plane Reynolds stress is very small, yielding a very small in-plane production of TKE. The opposite is seen in the inflows to exit row turbines. There $-\overline{u v} / U_{\text {hub }}^{2}$ is present but the wall-normal gradient of streamwise velocity is greatly reduced within the array.

From here, the discussion moves toward the anisotropy of turbulence and the invariants of $b_{i j}$ shown in Figures 11(c) and 11(d). According to the contour plots in Figure 11(c), inflows to the $\mathrm{CCW}$ rotating blades are slightly more anisotropic than the $\mathrm{CW}$ rotors as indicated by the increased values of $\eta$. This is seen especially in the inflow to the checkerboard case, where the minimum value of $\eta$ is approximately 0.05 , as opposed to the column-by-column case where $\eta$ reaches a minimum value of approximately 0.03 . Regardless of configuration, the minimum value of $\eta$ is still aligned with the hub in the far wake of the third row turbine. The turbines with a lesser magnitude of $P_{12}$ (uniform and checkerboard cases, CCW rotors) show greater values of $\eta$ in their inflows. This suggests that when a wind turbine experiences less isotropic turbulence in its inflow, the production of TKE is reduced in its wake. Truly isotropic turbulence, which requires $\eta=0$ is not reached in any of the cases.

In the wakes of the exit row turbines, $\eta$ shows generally the same trends as the entrance row wakes, although the magnitude of $\eta$ is greater. The maximum values of $\eta$ (least isotropic turbulence) trail the rotor at top-tip height. The region of small $\eta$ follows the nacelle and grows downstream of the turbines, indicating that the cores of the wakes are more isotropic and that the region of relative isotropy grows outward from the hub progressing with the main flow.

Looking to the inflow windows, $\xi$ varies considerably from case to case. The uniform and column-by-column arrangements show values of $\xi$ that are almost entirely positive or negative, respectively, in the measurement fields. These cases represent extremes of efficiency (discussed in Sec. IV E) and of anisotropy experienced by the turbines. The row-by-row case shows a mixture of positive and negative values of $\xi$ in the inflow arising from the alternation of rotor direction in upstream devices. The checkerboard arrangement is divided into a region of $\xi>0$ below hub height and $\xi<0$ above. From the wakes shown in Figure 11(d), the turbulence following the extremes of the rotors tends toward prolate spheroids and the characteristic spheroids following the nacelles of the turbines are dominantly oblate.

Another point of interest in the figure is that the $\xi<0$ region of the wakes drifts slightly upward or downward depending on the direction of rotation of the rotors. The uniform and checkerboard cases show a general downward drift progressing downstream of the turbines. The $\mathrm{CW}$ rotors show a slight upward drift of this region. A common feature of all measurement locations is that $\xi$ is always either significantly positive or negative in the near wake. This reinforces the point made above that the turbulence never reaches a truly isotropic state close to the wind turbines, where $\xi=0$ (and $\eta=0$ ) would be required.

The invariants plotted against one another in the AIM in Figure 12. As discussed in Table I, special cases of turbulence can be characterized by the two invariants of $b_{i j}$ at the theoretical limits of the Lumley triangle. Contours of $\eta$ and $\xi$ suggest that turbulence is organized into distinct regions within the wake as the flow moves away from the turbines.

In each of the anisotropy invariant maps in the Figure 12, invariants corresponding to the upstream window (black points) are more tightly grouped than those from the downstream measurement window (red points). This indicates that the anisotropy of turbulence is more uniform in the far wakes of the wind turbines. Figure 12 also indicates that there are both prolate and oblate characteristic spheroids in all measurement cases, according to the spread of points in the respective Lumley triangles. The exception to this trend is seen for the inflow the column-by-column case. There (black points in Figure 12(c)) the characteristic shapes overwhelmingly favor oblate spheroids, reflecting the negative values of $\xi$ seen in Figure 11(d). The complementary rotation of columns of turbines in the column-by-column case leads to a significantly different organization of turbulence than in other cases. Turbulence in the wake of the same case favors prolate spheroids, as in the other cases, but shows slightly higher values of $\eta$ and $\xi$, indicating a larger degree of anisotropy and stretching of spheroids than in the other cases. 


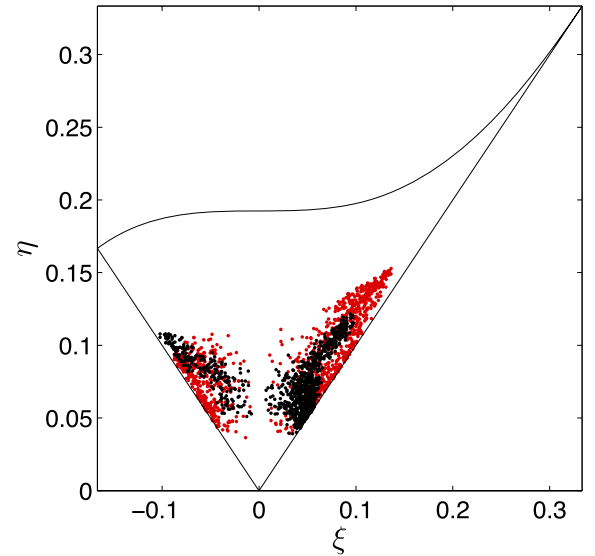

(a)

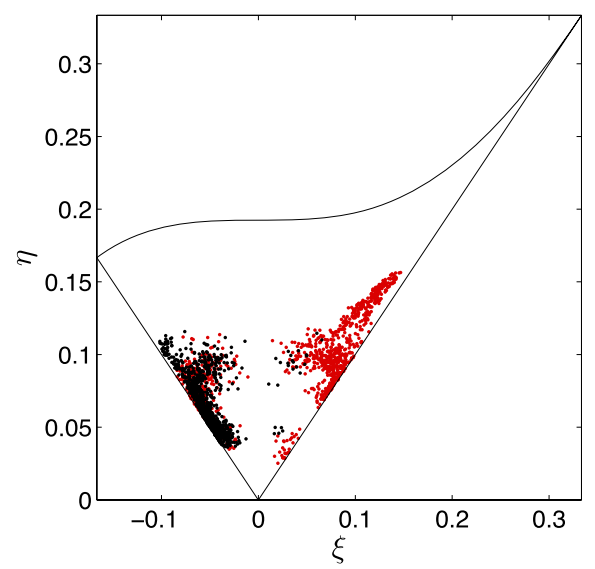

(c)

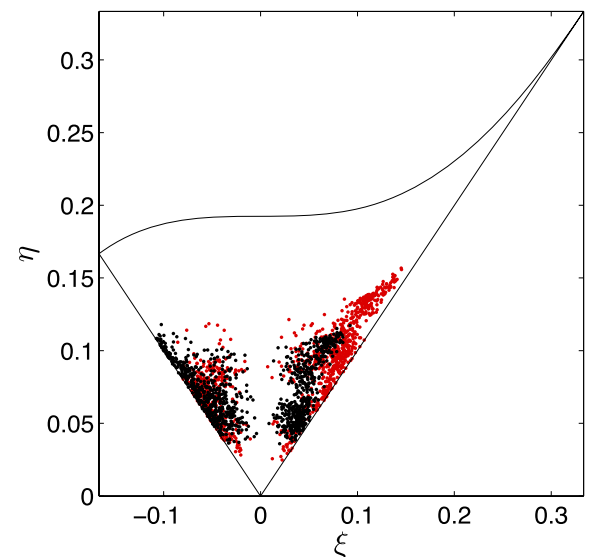

(b)

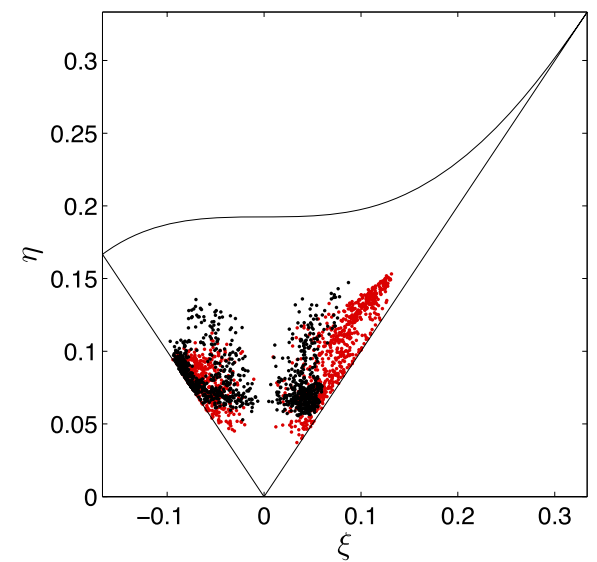

(d)

FIG. 12. Lumley triangles for each of the measurement cases. Points in black and red represent measurements directly upstream and downstream of exit row turbines. Every fifth point is plotted for clarity. (a) Exit row of the uniform arrangement. (b) Exit row of the row-by-row arrangement. (c) Exit row of the column-by-column arrangement. (d) Exit row of the checkerboard arrangement.

The inflow to the checkerboard case (Figure 12(d)) shows values of $\eta$ that are slightly greater than other cases. Values of $\xi$ are slightly less extreme than for the uniform and row-by-row cases, indicating that turbulence there is furthest from the idealized limits shown in the Lumley triangle. These behaviors are also seen in the contour plots of the invariants $\eta$ and $\xi$, Figures 11(c) and 11(d). Mentioned above, a greater magnitude of this invariant implies less isotropic turbulence. This indicates that the far wakes of turbines in the checkerboard arrangement are less isotropic than the other cases. Anisotropy is imparted to the flow by the alternation of rotational direction of the rotors in both the streamwise and spanwise directions, stopping the potential formation of large-scale streamwise structures in the column-by-column case.

\section{Rotation of principle coordinate system}

The Euler angles of rotation derived from the direct cosine matrix, $R$ (Eq. (8)) are shown in Figure 13. The three Euler angles $\theta_{x}, \theta_{y}$, and $\theta_{z}$ have been conditioned with a two-step filter discussed in the Appendix. In the leftmost column of the figure, there is a region of small rotation $\left(\theta_{x}<\pi / 4\right)$ following the nacelles for all cases. In the cases of CCW rotating blades (uniform and checkerboard cases, top and bottom rows, respectively), this region is less pronounced than for the $\mathrm{CW}$ cases. Following the rotor of the $\mathrm{CCW}$ cases at where $0.5 \leq y / D \leq 0.75$, the contours show rotations about the $x$-axis greater than for $\mathrm{CW}$ rotors. After the top-tip of the rotor, the region where 

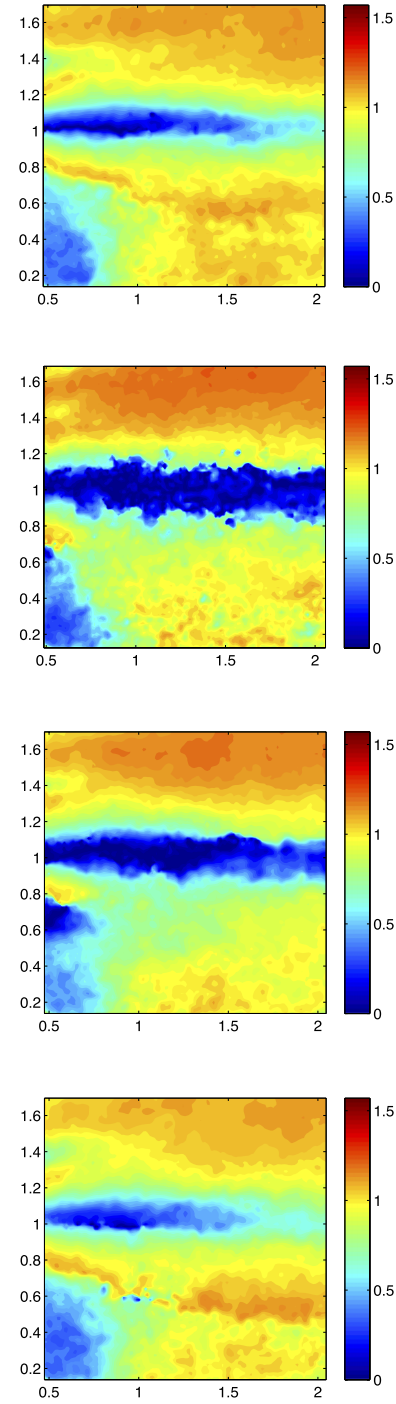

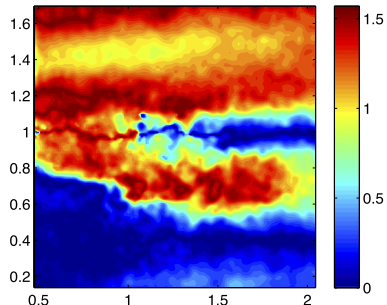

(a)

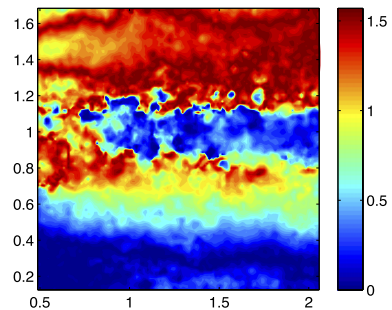

(b)

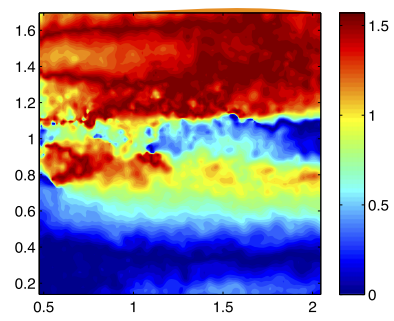

(c)

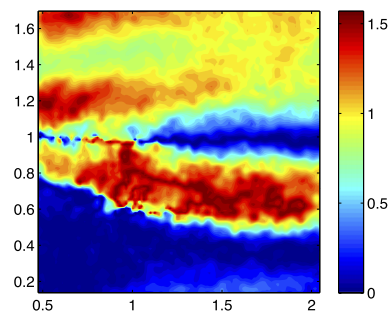

(d)
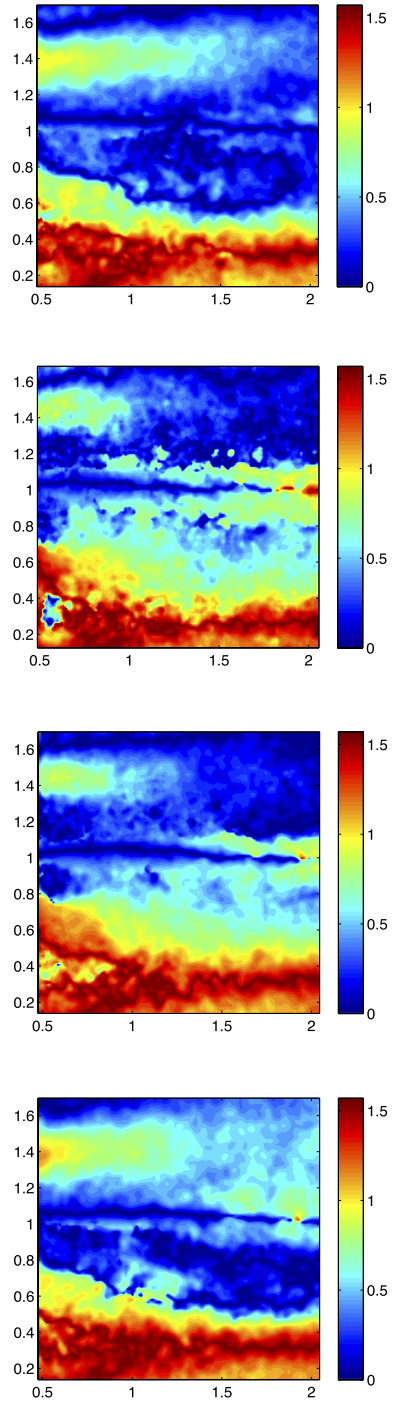

FIG. 13. Euler angles (about default axes) of rotation of principle coordinate system. Columns from left are $\theta_{x}, \theta_{y}$, and $\theta_{z}$ (rotations about the streamwise, wall-normal, and spanwise coordinates, respectively). (a) Uniform case. (b) Row-by-row case. (c) Column-by-column case. (d) Checkerboard case.

$\theta_{x}>\pi / 4$ roughly traces the shape of the in-plane Reynolds stress $-\overline{u v}$ and reflects the relative magnitude, greater for $\mathrm{CW}$ cases.

Clear differences between cases are seen in $\theta_{y}$ (center column of subfigures). In all cases, there are distinct regions following the hub where rotations are small. Moving both upward and downward away form the hub, every case shows that rotation of the principle axes about the $y$-axis tends toward greater values. The region of small rotations near the tunnel floor is accentuated in the $\mathrm{CCW}$ rotors compared to $\mathrm{CW}$ rotors. In the upper region of the wakes following the rotor at top-tip height, $\theta_{y}$ shows prominent differences based on direction of rotation of the blades. For CCW rotors, $\theta_{y} \approx \pi / 4$ and extends far into the wake. For $\mathrm{CW}$ rotors, $\theta_{y}$ reaches its maximum values of $\theta_{y} \approx \pi / 2$ following the top-tip of the rotor.

Regions of large rotation about the $z$-axis of the Cartesian system are localized to the bottom of the measurement domains and similar for all cases. The treated Euler angles show that for much of the measurement domain, $\theta_{z}$ is small compared to the other angles. There are noticeable differences 


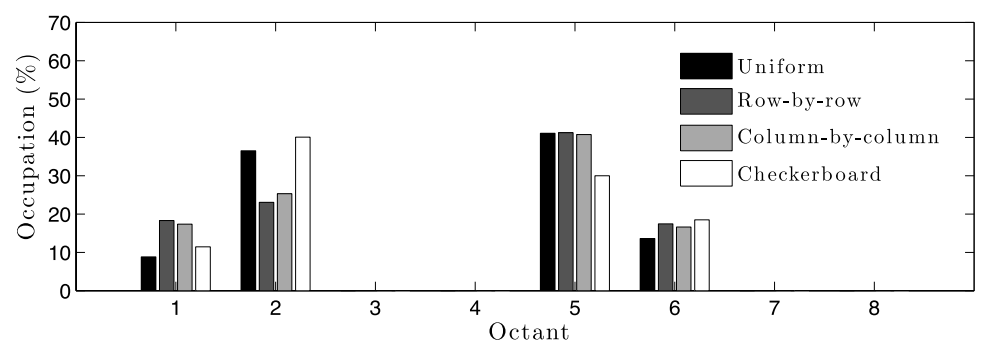

FIG. 14. Residence of characteristic vector $\overrightarrow{r^{\prime}}$ in original Cartesian octants after application of treatment to Euler angles.

between the cases based on direction of rotation, however. CCW rotors show an increase rotation about $z$ following the rotor at top-tip height, opposite of the trend seen for $\theta_{y}$.

To determine where the principle axes reside with respect to the original Cartesian system, the Euler angles of rotation are applied to the characteristic vector $\vec{r}$ defined in Eq. (9). The rotated vector $\overrightarrow{r^{\prime}}$ reveals in which octant, the principle coordinate system resides and is tallied for all measurement locations effectively combining the information in Figure 13. The relative occupation of $\vec{r}^{\prime}$ is shown in Figure 14 for the exit row wakes of all test cases. From the figure, it is clear that CCW rotors favor octant 1 over $\mathrm{CW}$ rotors; the opposite is seen for octant 2. Octants 5 and 6 are relatively equally occupied by all test cases.

Eigenvalues defining the radii of the characteristic spheroids discussed above and the principle axes defining their orientation both result from the decomposition in Eq. (5). The synthesis between the degree of anisotropy and alignment of the principle axes can be seen in Figure 15. As discussed pertaining to the spheroids shown in Figure 1, a truly isotropic state of turbulence is described by $\lambda_{i}=0$. Thus, smaller characteristic shapes shown in Figures 15(a) through 15(c) can be said to be more isotropic than ones with larger radii. The figure shows rotated characteristic spheroids in both inflow and wake locations for exit row turbine of all ray configurations. The uniform and column-by-column configurations of the wind turbine array represent the extremes of park efficiency discussed in Sec. IV E.

The flow visualization presented in Figure 15 contains the sum of information derived from the above analysis of anisotropy in wind turbine arrays. Discussing the particular balance of components in a tensor field presents some difficulty in that there are more elements than can typically be displayed in a single figure. Through the analysis here, the second-order Reynolds stress anisotropy tensor is essentially reduced to a first-order through the eigenvalue decomposition. However, this reduction requires a change in orientation of the given coordinate system in order to retain the information in the anisotropy tensor. Changes seen in the spheroids are directly related to the physical balance of stresses in the flow. Each spheroid communicates how many components are necessary to describe the stress field at a given location in the flow, one for prolate spheroids, two for oblate spheroids, and three for isotropic turbulence. The orientation of the spheroids indicates the direction in which these stresses are acting. That the principle vectors do not align with the default Cartesian coordinates of the wind tunnel is intuitive in that the turbulence here arises from a complex interaction of the $\mathrm{ABL}$ and the rotating geometry of the turbine rotors.

Inflow windows (left column) of Figure 15 demonstrate larger radii according to the values of $\eta$. In all cases, the characteristic spheroids get smaller approaching $y / D=1$. Spheroids in the inflow windows reflect the contour plots of $\xi$ where negative values correspond with oblate shapes and positive values correspond with prolate shapes. The inflow to the row-by-row case shows discontinuities in shape and orientation of the spheroids following the rough contours of $\xi$. In the inflow to the uniform and checkerboard arrangements, the spheroids near the top of the rotor are much larger than near the hub area, different from the column-by-column arrangement. Gradients in orientation of the spheroids are in the streamwise direction are less distinct than those progressing in the wall-normal direction.

Wake areas (right column) show expected variation in characteristic shapes and orientation of the anisotropy field combining information from the invariants of $b_{i j}$ from Figures 11(c) and 11(d) 

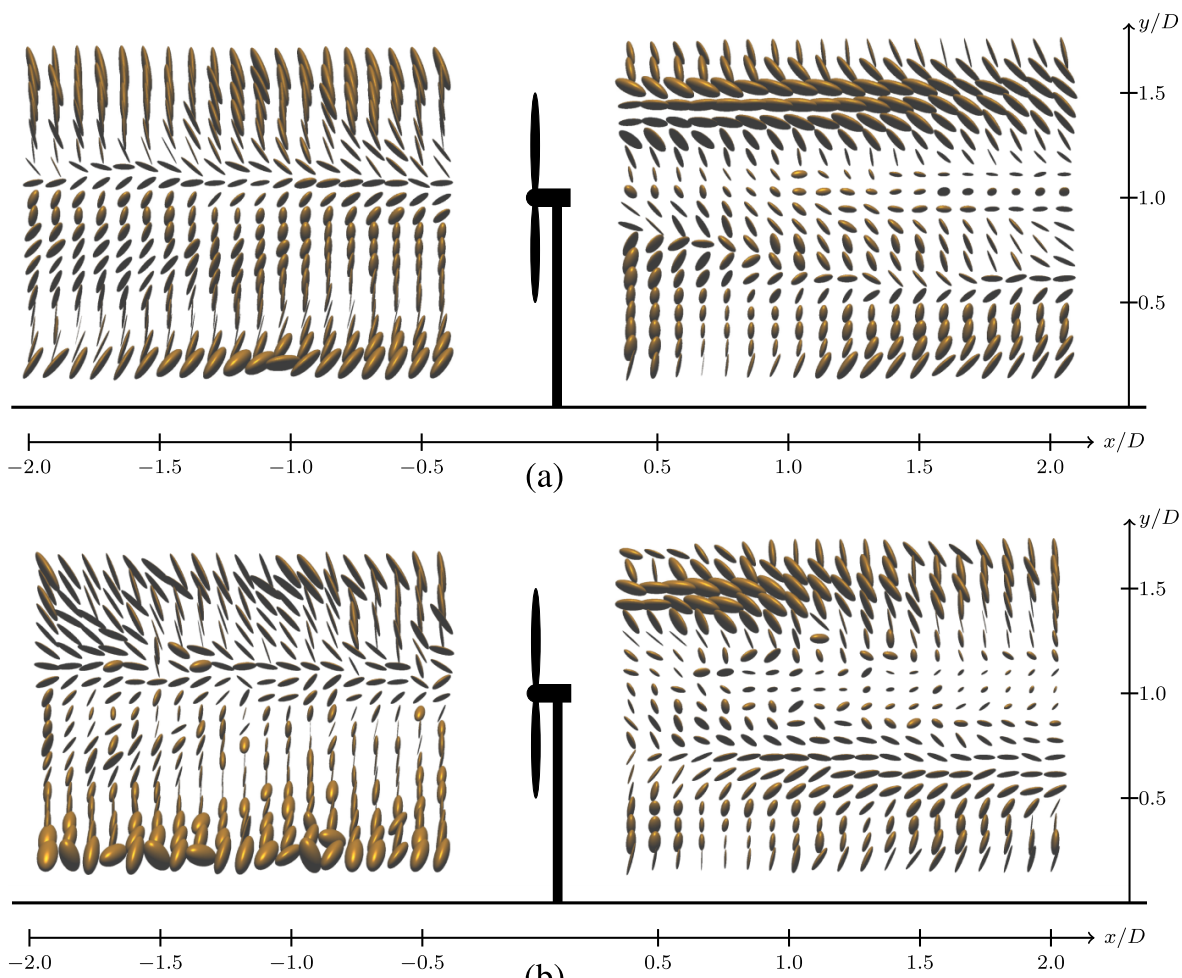

(b)

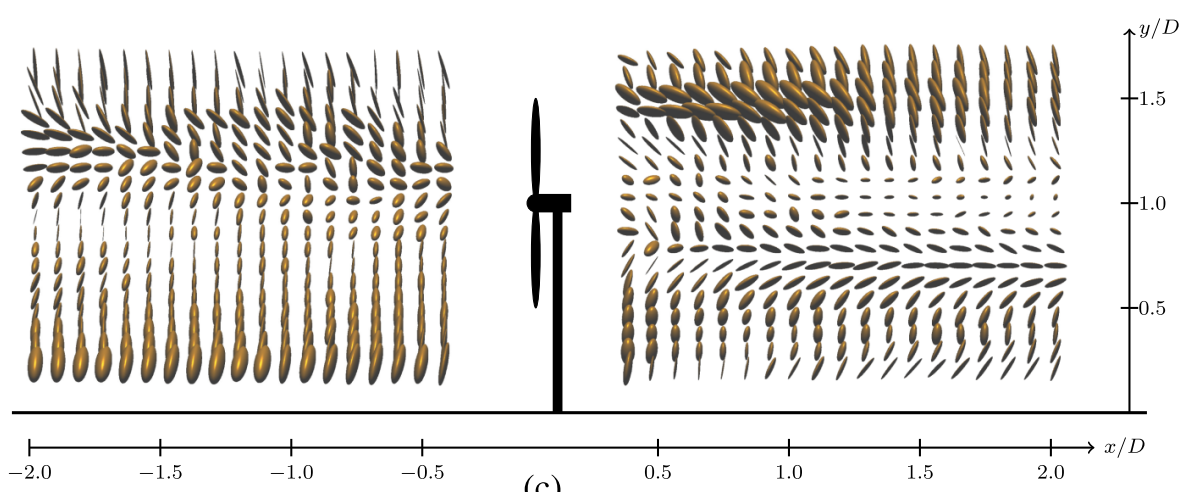

(c)
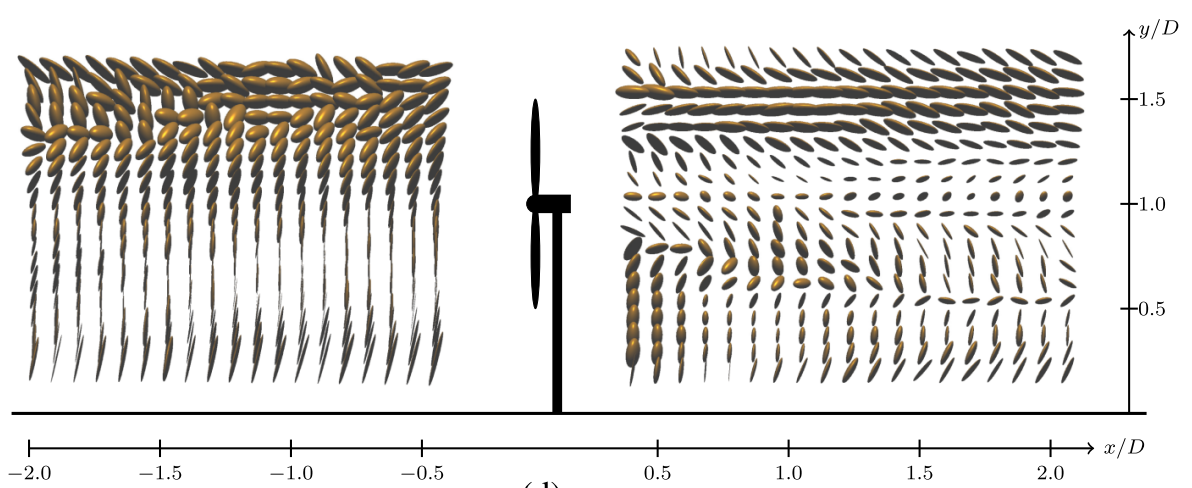

(d)

FIG. 15. Rotated characteristic spheroids for exit row turbine wakes. Fields are tilted along the $x$-axis to better illustrate spheroids. Only one hundredth of the total number of spheroids is plotted for clarity. (a) Rotated characteristic spheroids in the inflow and wake of the uniform arrangement. (b) Rotated characteristic spheroids in the inflow and wake of the row-by-row arrangement. (c) Rotated characteristic spheroids in the inflow and wake of the column-by-column arrangement. (d) Rotated characteristic spheroids in the inflow and wake of the checkerboard arrangement. 
and the filtered Euler angles from Figure 13. The most isotropic states of turbulence follow the hubs of the turbines after $x / D \approx 1.25$ in agreement with Figure 11(c). All four configurations of the turbine array demonstrate similar behavior in Figure 15 wherein there is a stretching of the spheroids (tending toward prolate shapes) trailing the top-tip of the rotor and near to the wind tunnel floor, and a flattening of spheroids (tending toward oblate shapes) following the central region of the wake.

The rotations of the spheroids in Figure 15 show dependence on the rotational sense of the rotor blades. CCW rotors (uniform and checkerboard cases) show a larger region of spheroids rotated about the $z$ - and $y$-axes than do CW rotors (row-by-row and column-by-column cases) following the rotor at top-tip height, echoing the contours of Figure 13. The rotation about the $y$-axis is evident in the wakes by the shading of the spheroids. Below hub height where $\theta_{y}$ is minimum just below the hub and increasing downward, the spheroids show their well lit sides and are lighter in color. The opposite is seen above the hub, where the spheroids have been rotated up to $\pi / 2$ radians about the $y$-axis.

\section{Flux and production}

The flux of kinetic energy $F_{12}$ shown in Figure 11(a) quantifies the kinetic energy from the flow above the turbine canopy transported by large-scale turbulence into the momentum-deficit area of the wake. In its complete formulation, the flux term is second-order tensor multiplying the mean velocity and the Reynolds stress tensor.

Comparing Figures 11(a) and 11(d), regions in which $F_{12} \geq 0$ correspond well with regions of the wake in which $\xi \geq 0$. A demonstration of this is seen in Figure 16 wherein a threshold was set based on the algebraic signs of each quantity (left and center columns of the figure, respectively). Viewing the two quantities in this way makes determining their overlapping regions clear. The right column of subfigures indicates the correlation of the two signs of the quantities. For the overlap in the right column, white and black areas indicate that the signs of $F_{12}$ and $\xi$ are the same behind the exit row turbines and either positive or negative, respectively. The areas where the signs of the two quantities are not the same are shown in gray. From the figure, it can be seen that the match of signs is more prevalent in the uniform and checkerboard cases (CCW rotation) than it is in the row-by-row or column-by-column cases ( $\mathrm{CW}$ rotation). The difference in overlap can be attributed mainly to the sign of $\xi$, as the contours denoting the algebraic sign of $F_{12}$ are very consistent between cases.

Comparing only the algebraic signs of $F_{i j}$ and $\xi$ disregards the magnitudes of either quantity, yet the overlap regions indicate strong behavioral tendency in the wakes of wind turbines. A positive value of $F_{i j}$ indicates a downward flux of kinetic energy into the wake and a negative value indicates an upward flux. Coupling this with the sign of $\xi$ indicates that when the flux of kinetic energy is downward, it is overwhelmingly associated with turbulence whose characteristic spheroids tend to be prolate and exhibit a single eigenvalue larger than the other two. Likewise, upward flux is coupled with turbulent structures forming oblate spheroids and exhibiting one eigenvalue smaller than the other two. Stated otherwise, downward flux is associated with turbulence with one dominant component of the anisotropy tensor and upward flux is associated with two co-dominant components.

The regions of Figure 16 denoted by gray contours are areas in which the direction of flux and the shapes of the characteristic spheroids have the opposite association. Gray areas above the hub $(y / D>1)$ are oblate spheroids and contributing to downward flux. Below the hub $(y / D<1)$, the gray denotes prolate spheroids associated with upward flux. There is a consistent pattern moving upward through the overlap contours (white, gray, black, gray, white). This pattern suggests that the relationship between $F_{12}$ and $\xi$ is highly dependent on $y / D$. According to overlapping threshold maps, the mismatch between the signs of the flux of kinetic energy and $\xi$ is more pronounced in the wakes following the $\mathrm{CW}$ rotors. As the thresholds for the flux of kinetic energy are very consistent from case to case, this difference can be mainly attributed to $\xi$. The upward drift of $\xi<0$ for the $\mathrm{CW}$ cases increases the mismatch region from approximately $10 \%$ of the window for $\mathrm{CCW}$ wakes to approximately $35 \%$. 

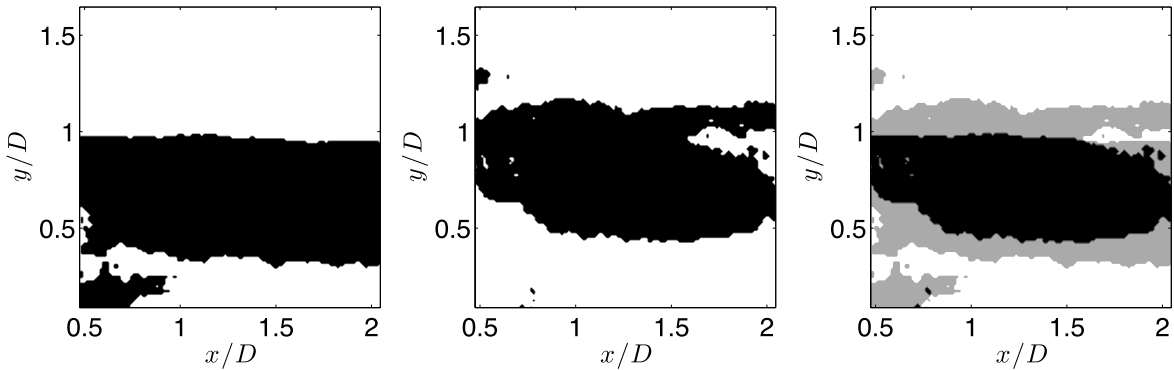

(a)
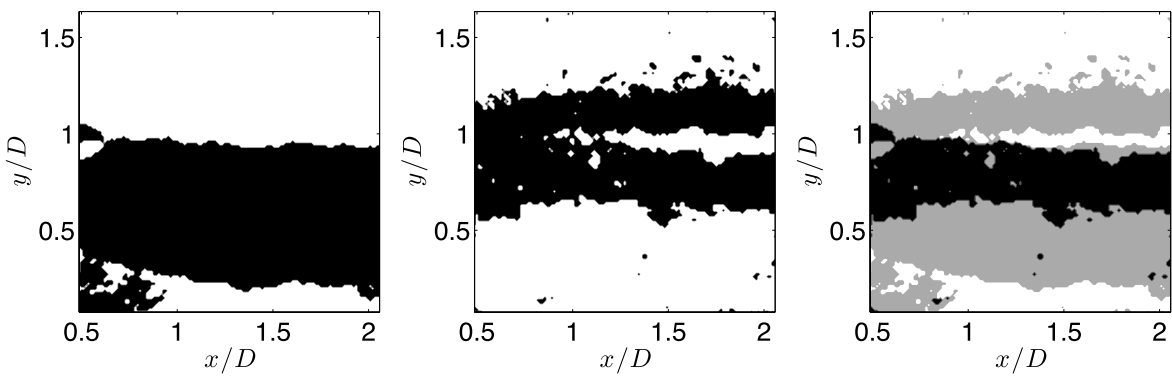

(b)
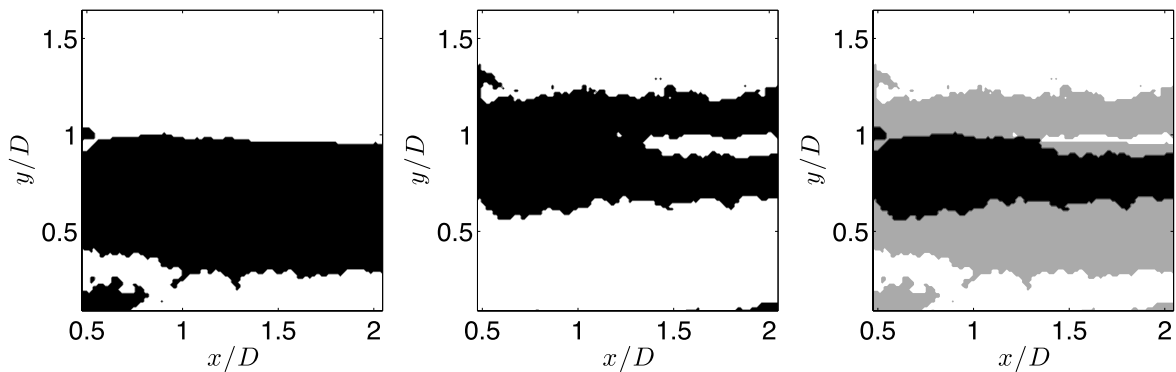

(c)
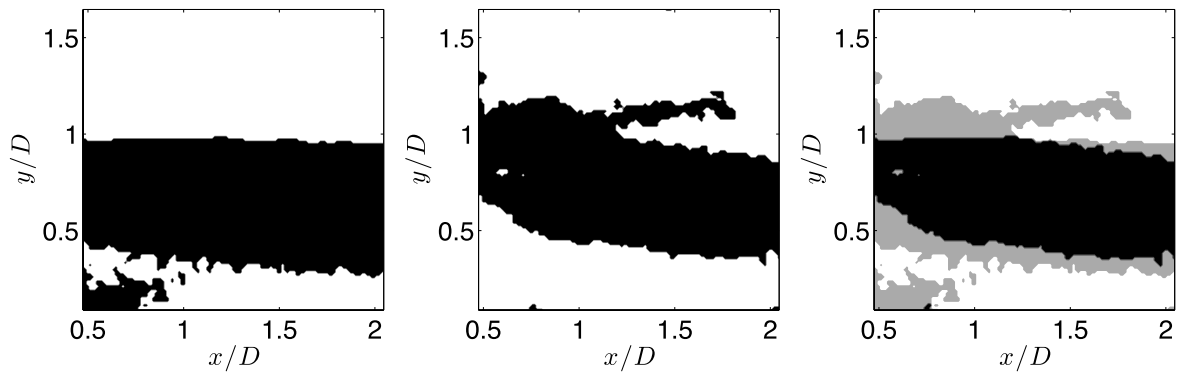

(d)

FIG. 16. From left are the algebraic signs of $F_{12}$ and $\xi$ and their regions of overlap. In the right column, overlaps are positive or negative and matching (in white and black, respectively) or not matching (in gray). (a) Uniform case. (b) Row-by-row case. (c) Column-by-column case. (d) Checkerboard case.

A similar approach is taken for the in-plane production of TKE, $P_{12}$, and the second invariant of the normalized Reynolds stress tensor $\eta$. The magnitude of $\eta$, ranging from 0 to $1 / 3$, is an indication of the anisotropy of turbulence. As with the flux, $P_{12}$ includes the Reynolds stress tensor in its full composition and as such, a connection between $P_{12}$ and the invariants is anticipated.

In Figure 17, the quantities compared $\left(P_{12}\right.$ and $\left.\eta\right)$ are positive by definition, and the comparison made in Figure 16 is inappropriate. Noting that in the contour plots of $P_{12}$ and $\eta$ (Figures 11(b) and $11(\mathrm{c}))$, there is a correlation between the regions of relatively high production and larger values of $\eta$. In the left column of Figure 17, values of $P_{12}$ less than $1 \%$ of the maximum are shown in black and considered to be regions of low production. The threshold for $\eta$ was set to the mean value in each of 

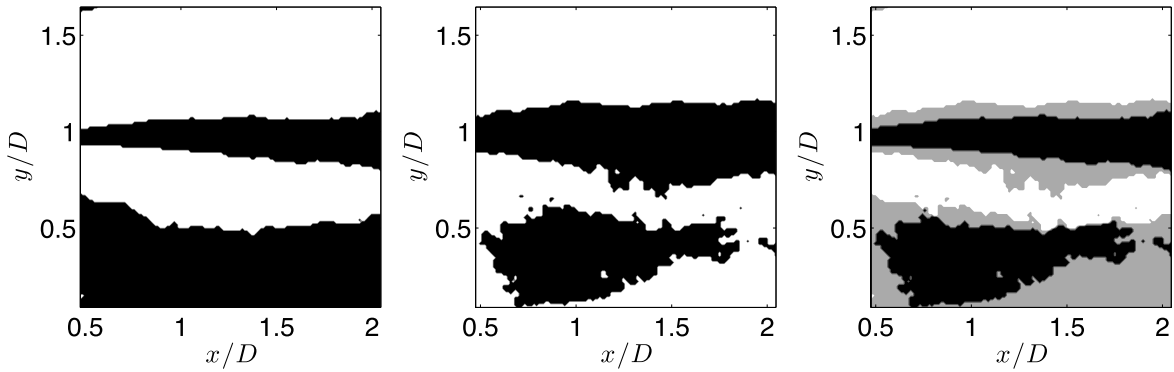

(a)
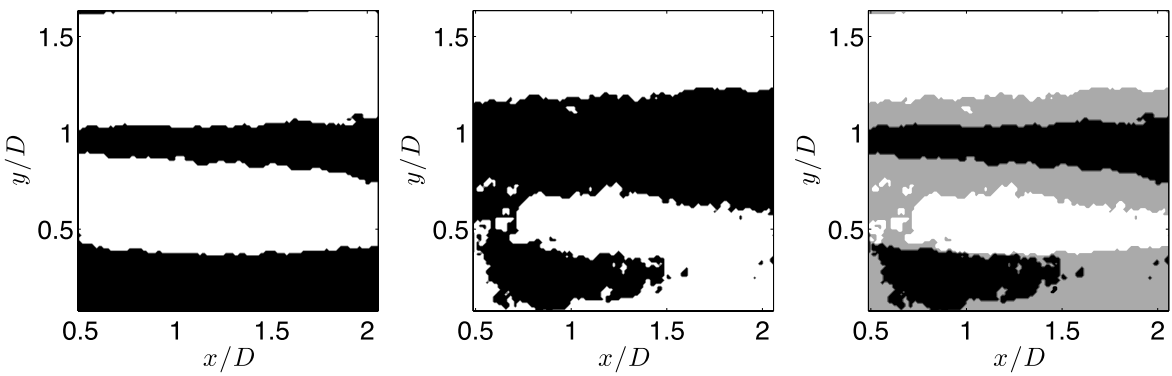

(b)
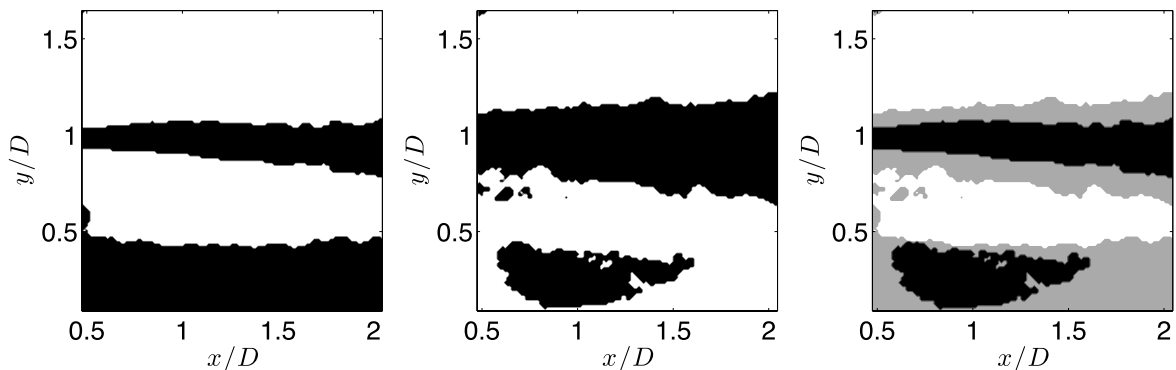

(c)
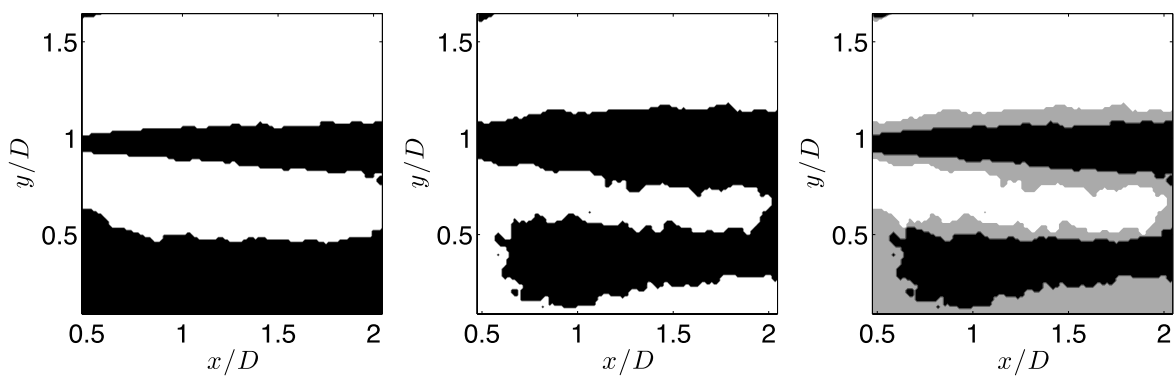

(d)

FIG. 17. Threshold plots of $P_{12}$ and $\eta$ in the left and center columns, respectively. The right column shows overlapping areas as high or low matching values according to the thresholds (in white and black, respectively) or not matching (in gray). (a) Uniform case. (b) Row-by-row case. (c) Column-by-column case. (d) Checkerboard case.

the wakes and roughly classifies the anisotropy seen in the wakes as either "weak" or "strong" but does not result from an obvious division of values.

The overlap of the threshold conditioned production and second invariant indicate that there is almost total agreement in the region of the wake following the top-tip of the rotor. This indicates that the productive regions (in the sense of TKE) correlate with regions of anisotropic turbulence; i.e., vertical gradients in the mean flow are associated with productive and anisotropic regions in the wakes. The overlap maps in the right column of Figure 17 also show that the central region of the wake (following the hub) are regions of low production and that the turbulence there is much more isotropic. 


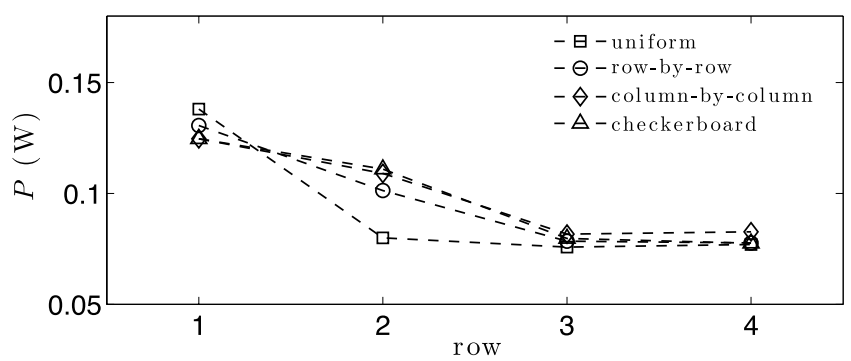

(a)

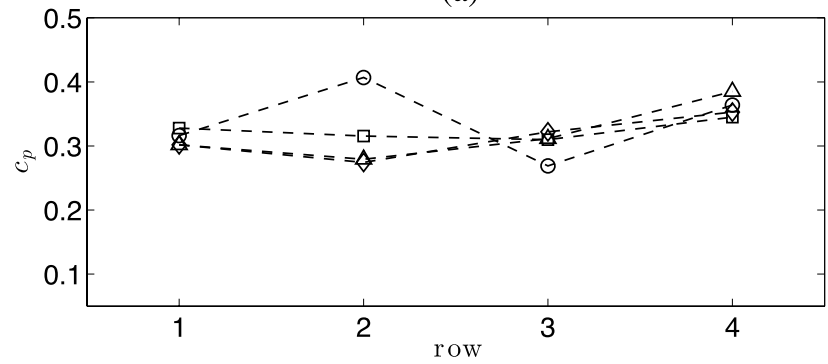

(b)

FIG. 18. Development of power and efficiency by wind turbines in the four configurations of the wind turbine array. (a) Peak power produced by center column turbines by row. (b) Power coefficient for center column turbines by row.

The significance of the gray mismatch areas in Figure 17 is less clear than in the overlap of $F_{12}$ and $\xi$. In the right column of subfigures, the gray contours following the hub $(0.75<y / D<1.25)$ correspond to high production but a lesser degree of anisotropy. In the area of the wake below the rotor $(y / D<0.5)$, gray contours are areas of low production and more anisotropic flow. The contours of $\eta$ in Figure 11(c) indicate that the transition between weak and strong is continuous. Nevertheless, the overlap of the thresholds is consistent with matching areas taking up approximately $75 \%$ of the wake areas. The overlapping thresholds of terms from the mean mechanical energy equation and the invariants of the normalized Reynolds stress anisotropy tensor (Figures 16 and 17) are sufficient to demonstrate that there are correlations between the quantities, although the functional relationship between all quantities is not undertaken in this work. As was the case for the flux and third invariant, the thresholds of production in the left column are very consistent from case to case. The mismatching areas in the right column can then be mainly attributed to differences in the threshold of $\eta$. The wakes following CW rotors (center two rows) show less agreement of $P_{12}$ and $\eta$ below $y / D=0.5$ than do the CCW rotors. Because the threshold of production is very similar for all cases, this difference is mainly attributed to the region of $\eta<\eta_{\text {mean }}$ which is smaller for $\mathrm{CW}$ rotors. Conversely, near hub height of the wakes, the $\mathrm{CW}$ rotors show a much larger area of $\eta<\eta_{\text {mean }}$, indicating that the core of the wakes tends to be more isotropic than for CCW rotors. This is confirmed by the contours of $\eta$ in Figure 11(c).

\section{E. Power and efficiency}

Important considerations with any wind energy experiment are the power generation and efficiency of the devices in the array. Figure 18 shows the power and power coefficient of center column devices in each of the four array configurations. Entrance row turbines produce between 0.13 and $0.14 \mathrm{~W}$. The expected decrease in productivity of turbines is evident in all four configurations to varying degrees, but most prevalent in the uniform arrangement, where the power produced drops sharply in the second row. The productivity of each array configuration is very similar in the third and fourth rows. A standard Cartesian array is considered fully developed in terms of periodic wake dynamics by the fourth row, indicating an asymptotic power production of approximately $0.076 \mathrm{~W}$. The increased productivity of the test configurations makes the fully developed assertion difficult to make although the results in Figure 18(a) indicate that the fourth row may be near the asymptotic production. 
Looking to the power coefficient in Figure 18(b), it is evident that entrance, third, and fourth row devices have similar efficiencies between cases. In the second row, where devices have considerable range of power production, there is also a large spread of $c_{p}$. There the row-by-row case exhibits $c_{p} \approx 38 \%$, the highest value of any measurement. Exit row turbines show two distinct efficiencies based on rotational sense of the turbines. There CCW rotors have and efficiency of approximately $33 \%$ and $\mathrm{CW}$ rotors operate at approximately $25 \%$. The measurement error for power and power coefficient is estimated to be within $6 \%$. At this level, the differences in $c_{p}$ are distinct although the cause of this difference is not undertaken here.

Figure 19(a) shows the mechanical power for exit row turbines versus angular velocity of the rotor. From the shown curves, it is evident that the exit row turbine of the checkerboard arrangement produces a peak power approximately $5 \%$ greater than the other cases. The angular velocity corresponding to this peak is also lower than for the other arrangements, occurring at roughly $140 \mathrm{rad} / \mathrm{s}$ rather than between 180 and $200 \mathrm{rad} / \mathrm{s}$. Similarity in the power curves between cases indicates that the mean velocity in the inflows seen from case to case is also quite similar. Figure 19(b) shows curves of the power coefficient and indicates that models operate near $35 \%$ efficiency. There is a disparity of approximately $6 \%$ between the best (column-by-column) and worst (uniform) performers in the exit row. Peaks of the power coefficient occur at the tip speed ratio of $2.35 \leq \lambda_{t s r} \leq 3.25$, low in terms of real devices but reasonable for wind tunnel experiments. ${ }^{35}$

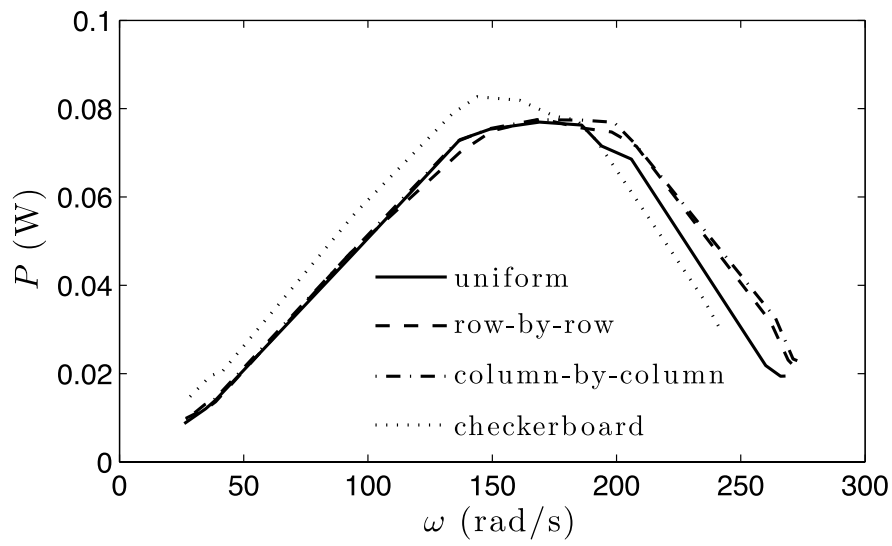

(a)

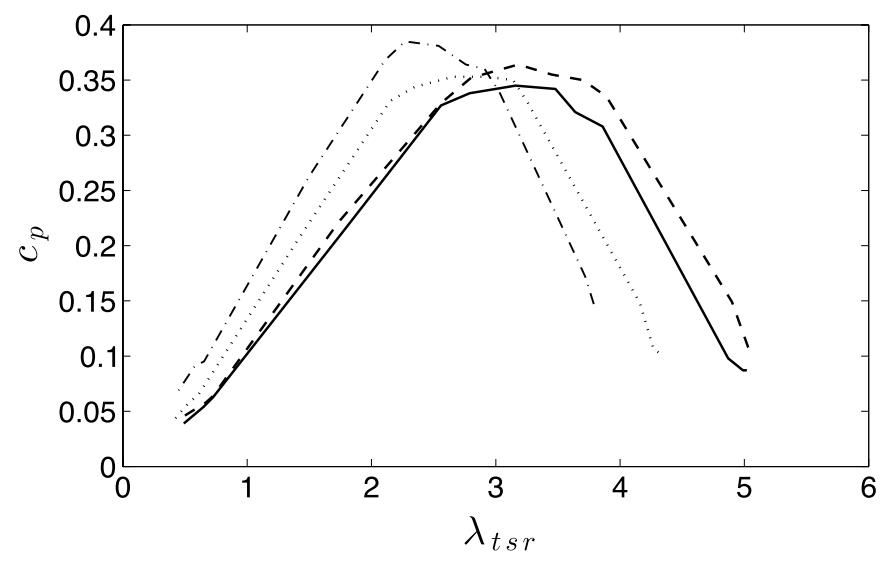

(b)

FIG. 19. Power curves and curves of power coefficient for all exit row turbine models. (a) Power curves for exit row turbines. (b) Curves of power coefficient for exit row turbines. 
TABLE II. Park efficiency by array configuration.

\begin{tabular}{lc}
\hline \hline Configuration & $\zeta(\%)$ \\
\hline Uniform & 67 \\
Row-by-row & 72 \\
Column-by-column & 80 \\
Checkerboard & 78 \\
\hline \hline
\end{tabular}

To distinguish the performance of each test array the park efficiency is considered according to the work by Kang and Meneveau. ${ }^{30}$ The efficiency of a wind farm can be evaluated as,

$$
\zeta=\frac{P_{\text {real }}}{P_{\text {ideal }}} \times 100 \%,
$$

where $P_{\text {real }}$ is the sum of power produced by all devices in an array and $P_{\text {ideal }}$ is what the power would be if each device behaved as though it was in an entrance row. The park efficiency for the each configuration of wind turbines analyzed here is listed in Table II.

These data indicate that although exit rows produce approximately the same power, the array configurations behave differently from case to case. The increase in $\zeta$ for counter-rotating cases over that of uniform rotation is attributed to an increase of lift on the rotor blades due to agreeable rotation in the mean velocity and large scale turbulence acting as inflows for non-leading turbines. Of the non-uniform cases, the checkerboard and column-by-column cases demonstrate the greatest increase in park efficiency. From Figure 18(a), it is evident that the main boost in park efficiency comes from differences in production of second row devices.

The increases in these cases indicate that there is wake interaction crossing streamwise columns of a wind farm. The influence of neighboring wakes is significant to park efficiency quite quickly, within the first few rows. As seen in the AIMs of the four test configurations in Figure 12, the column-by-column arrangement shows an inflow to the exit row turbine that is significantly more uniform that the other cases. This difference is due to the development of counter-rotating structures on the scale of the spanwise turbine spacing $S_{z}=3 * D$ seen in Figure 20

Comparing these values of $\xi$ from the contour plots in Figure 11(d) to the park efficiencies in Table II indicates that negative values of $\xi$ augment park efficiency. This suggests that increasing the power of wind turbines and arrays may be aided by conditioning the turbulence in the inflow. A negative correlation between $\zeta$ and $\xi$ would suggest that turbulence that is squeezed in one eigenvalue (oblate characteristic spheroids) tends to increase overall wind park efficiency.

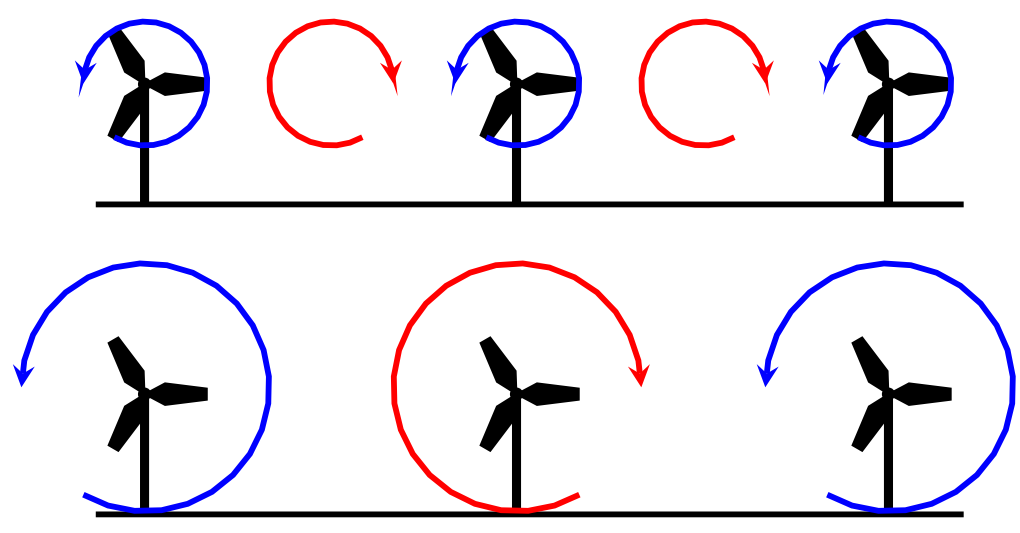

FIG. 20. Depiction of counter-rotating structures developing between rows of wind turbines. The case of uniform rotation (top) limits the size of structures to that of the wind turbine rotor. In the column-by-column case, the structures may grow to the size of the spanwise turbine spacing $S_{z}=3 * D$. 


\section{CONCLUSIONS}

The present study uses a Cartesian array of wind turbines assembled in the wind tunnel at PSU. Mean velocities show expected trends in the streamwise direction $U$ where momentum deficits in the wakes are clear, and the spanwise direction $W$ where the rotational sense of the rotors is distinguished. The rotation of the turbine rotors is also pronounced in the Reynolds shear stress $-\overline{v w}$. The spanwise spacing of devices within the model array was fixed at $S_{z}=3 * D$ which is smaller than that of many existing wind farms. The organization of the flow between successive rows of wind turbines and the increase of park efficiency seen in the column-by-column case likely arise from symmetry in the flow between the wind turbines in each row. Dynamical effects introduced by counter-rotating schemes in the experiment may be augmented through the decreased spacing between devices but are expected to prevail at larger spanwise spacings.

The normalized Reynolds stress anisotropy tensor $b_{i j}$ is composed from the turbulent stress tensor $\overline{u_{i} u_{j}}$ and the turbulent kinetic energy $k$. The second and third invariants of the tensor $\eta$ and $\xi$ are compared on a case-by-case basis. Although the differences of the invariants observed in the wakes are subtle, the wakes of exit row turbines show some structural dependency of invariants on the rotational sense of the turbine rotors. In the cases of counter-clockwise rotation of the rotor blades, the region of $\xi<0$ in the wakes drifts downward from the hub towards $y / D=0$. The opposite trend is seen for the counter-counter clockwise rotating blades. Extending the analysis of anisotropy through eigendecomposition of the normalized Reynolds stress anisotropy tensor yields a set of eigenvalues $\lambda_{i}$ and corresponding orthogonal vectors $\Sigma_{i j}$. The eigenvalues represent principle components of $b_{i j}$ at each point within the measurement windows and are used to compose spheroids characteristic of the turbulence in the turbine wakes of the exit row. Analysis reveals significant dependence of characteristic spheroids on the wall-normal coordinate in the wake. Spheroids show distinct character as oblate and prolate spheroids following bottom- and top-tips of the rotors, respectively. Following the hub, the spheroids favor oblate shapes and have smaller radii, corresponding to increased isotropy in that area.

The set of vectors associated with the principle components form an orthonormal basis and can be treated as a direct cosine matrix. From the direct cosine matrix, a set of Euler angles of rotation about the Cartesian coordinate axes are calculated. The orientation of these axes in the wakes depends on the rotational sense of the turbine rotors. A treatment was applied to the Euler angles of rotation, clarifying behavior in exit row turbine wakes. Rotations around the $y$-axis demonstrate minimum values directly following the nacelle in the wakes and increasing outward. The rotational sense of the rotors is clearly distinguished in $\theta_{x}$ following the nacelle and in $\theta_{z}$ following the top-tip of the rotor.

The normalized Reynolds stress anisotropy tensor is mapped in its principle components as a field of spheroids rotated about their respective centers. Comparisons of array configurations demonstrate similar behavior and confirms that turbulence trailing the rotor at top-tip height tends to exhibit one large eigenvalue and a prolate characteristic spheroid. Conversely, turbulence trailing the rotors at bottom-tip height demonstrates a single small eigenvalue and oblate characteristic spheroids. Noting that perfectly isotropic turbulence requires all eigenvalues to be identically zero, smaller characteristic spheroids are related to more isotropic behavior. In all four measurement cases, the size of the spheroids decrease distinctly as they approach the part of the wake following the nacelle, although they never reach radii equal to zero. The exit row turbine of the uniform case shows less isotropy of turbulence in the measurement areas than the column-by-column case, as indicated by the larger spheroids in the fields.

The areas of more isotropic turbulence, indicated by smaller spheroids, are more prevalent in array configurations where the rotational sense of the rotors changes in the spanwise direction. The inflow to the uniform case demonstrates $\xi>0$ nearly everywhere, associated with the lowest value of park efficiency. Conversely, the column-by-column arrangement shows values of $\xi$ that are dominantly negative. The decrease of turbulence indicates an overall organization of turbulence for the column-by-column arrangement of turbines. The complementary rotation of devices in the spanwise sense leads large counter-rotating structures on the order of magnitude of the spanwise 
turbine spacing. The row-by-row and checkerboard arrangements show a mixture of positive and negative values of $\xi$ in the inflow and are the middle performers from the current study.

In order to establish connections between the flux of kinetic energy $F_{12}$ and the third invariant of $b_{i j}$, threshold maps were made according to their respective algebraic signs and overlaid. The overlapping regions indicate positive correlations between the two quantities with mismatched signs tending to occur in the hub regions of the wake and near the bottom-tips of the rotors. This relationship implies that when the flux of kinetic energy is vertically downward, the turbulence tends toward stretched characteristic spheroids. Conversely, when the flux of kinetic energy is vertically upward turbulence is characterized by compressed spheroids.

A similar comparison was made between the production of TKE, $P_{12}$, and the second invariant of $b_{i j}$. Both of these quantities are by definition positive, making thresholds somewhat arbitrary. For the production, a threshold was selected at $1 \%$ of the maximum value for each wake. The threshold for $\eta$ was set at the mean value from each measurement window. Overlaying the threshold maps as above demonstrates that the production of TKE is correlated with anisotropic turbulence. The regions of overlap agree quite well for each exit row turbine wake, regardless of direction of rotation. Both quantities are associated with regions of mean velocity gradients in the wall-normal direction.

The results of the current analysis are applicable to wind farm simulation and planning software. The flux of kinetic energy and production of TKE correlate with the invariants of the normalized Reynolds stress anisotropy tensor indicating that anisotropy cannot be neglected in turbine array design. Increasingly, complex arrays are being designed through software with sparing site measurement to mitigate costs. A more complete understanding of possible optimizations of turbine arrays is required to rely on this method of design. The results here establish a basis for comparison or validation for future simulations incorporating rotational sense to array designs.

\section{ACKNOWLEDGMENTS}

The authors would like to extend their gratitude to the National Science Foundation BRIGE (Project No. ECCS-1032647) and IGERT (Project No. 0966376) programs for funding this research. The authors also acknowledge Mr. Dominic DeLucia for carrying out the torque measurements for the arrays. It is hoped that the results presented here may be reproduced and extended through future simulation and experimentation. To this end, the exchange of data with other researchers is encouraged.

\section{APPENDIX: FILTERING OF EULER ANGLES}

As discussed briefly in Sec. II, the output from the eigenvalue decomposition in Eq. (5) is a set of values corresponding to the principle components of the anisotropy tensor oriented along an orthonormal vector basis. The vector basis can be considered the optimal coordinate system spanning the vector space of the anisotropy tensor. Further, if the vector basis is concatenated, it can be considered as a direct cosine matrix, denoted here as $R$. Equation (A1) defining $R$ is repeated from Sec. II for ease of reading.

$$
R=\left[\begin{array}{ccc}
\cos \theta_{y} \cos \theta_{z} & \cos \theta_{y} \sin \theta_{z} & \sin \theta_{y} \\
\cos \theta_{x} \sin \theta_{z}+\cos \theta_{z} \sin \theta_{x} \sin \theta_{y} & \cos \theta_{x} \cos \theta_{z}-\sin \theta_{x} \sin \theta_{y} \sin \theta_{z} & -\cos \theta_{y} \sin \theta_{x} \\
\sin \theta_{x} \sin \theta_{z}-\cos \theta_{x} \cos \theta_{z} \sin \theta_{y} & \cos \theta_{z} \sin \theta_{x}+\cos \theta_{x} \sin \theta_{y} \sin \theta_{z} & \cos \theta_{x} \cos \theta_{y}
\end{array}\right] .
$$

The direct cosine matrix is composed of the three angles of rotation required to map the default Cartesian axes onto the principle axes of the anisotropy tensor. The Euler angles $\theta_{x}, \theta_{y}$, and $\theta_{z}$ are rotations about the $x, y$, and $z$-axes, respectively. The Euler angles are extracted from $R$ 


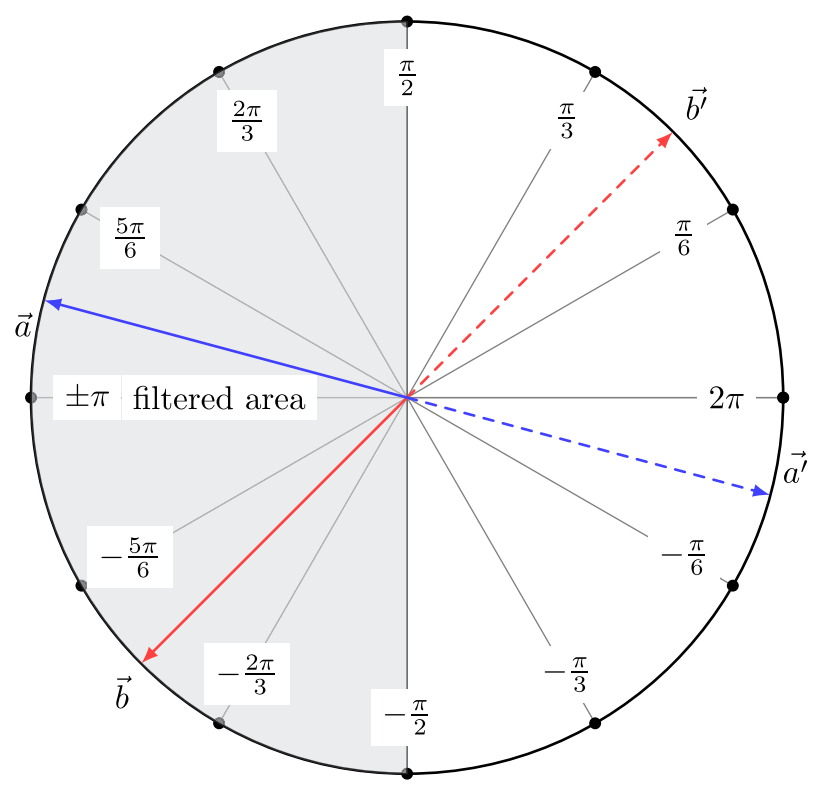

FIG. 21. Schematic detailing behavior of the filters applied to Euler angles. Vectors in the filtered areas (denoted without prime) are mapped across the origin. Filtered vectors are denoted with primes.

according to

$$
\begin{aligned}
& \theta_{y}=\sin ^{-1}\left(R_{1,3}\right), \\
& \theta_{x}=\cos ^{-1}\left(\frac{R_{3,3}}{\cos \theta_{y}}\right), \\
& \theta_{z}=\cos ^{-1}\left(\frac{R_{1,1}}{\cos \theta_{y}}\right) .
\end{aligned}
$$

The data for the Euler angles show discontinuities throughout the measurement fields resulting from the discrete nature of the measured data, numerical eigenvalue decomposition, and the bounded domain of inverse sine and cosine functions. The decomposition outlined in Eq. (5) seeks the most efficient vector basis for the turbulent stress tensor rather than the continuous changes from point to point, and thus is subject to some error when converted into Euler angles. The default output intervals for $\theta_{x}, \theta_{y}$, and $\theta_{z}$ derived from the direct cosine matrix in Eq. (8) are from $-\pi$ to $\pi$, leading to discontinuities in the rotations of the principle axes shown in Figure 22(a) when $\theta_{i} \approx \pm \pi$. Figure 22(a) shows the Euler angles extracted directly from $R$. From the contour plots of $\theta_{x}, \theta_{y}$, and $\theta_{z}$, it is clear that there are discontinuities in the plots whenever an angle crosses the periodic threshold at $\pm \pi$.

To clarify the rotations of the principle axes, a treatment was applied to the Euler angles. Any set of three orthonormal vectors can describe many equally efficient coordinate systems, allowing for permutation of $x_{i}$ and ignoring algebraic signs. Considering this, any Euler angle outside of a chosen range $\theta_{i}>\pi / 2$ or $\theta_{i}<-\pi / 2$ was mapped across the origin. Figure 21 illustrates the filtering of two example angles. However, under multiple rotations (successively about the three Cartesian axes), a vector under these filters can still be mapped into an undesirable region. To correct this, a final step in filtering was to take the absolute values of the three Euler angles. Contour plots of the Euler angles under the two-step filters are shown in Figure 22(b).

Validation of the filtering described above is shown in the residence of the characteristic vector $\vec{r}$ introduced in Sec. II. The residence of $\vec{r}$ rotated by all three Euler angles was tallied in each measurement area, shown in Figure 23(a). The figure shows that in the each measurement domain, the principle coordinate system of the anisotropy tensor is dominantly found in octants $1,2,5$, and 6 indicating that $\theta_{x}<\pi / 4$. The total (spherical) rotation of $\vec{r}$ is nearly always ( $\left.\sim 95 \%\right)$ constrained 

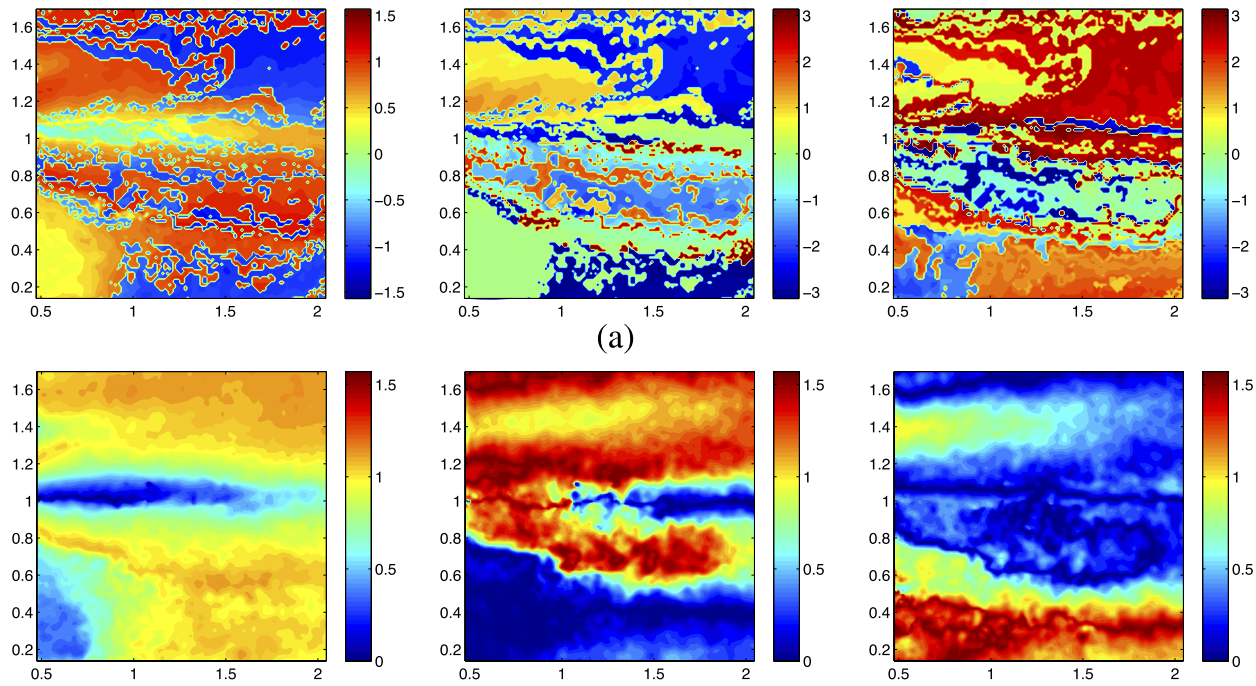

(b)

FIG. 22. Euler angles of rotation about the Cartesian axes. Data pertain to the wake area of the exit row in the uniform case. From left to right are rotations about the $x, y$, and $z$ axes; $\theta_{x}, \theta_{y}$, and $\theta_{z}$, respectively. (a) Raw Euler angles prior to application of filters. (b) Contours of the filtered Euler angles.

to the interval $-\pi / 2<\theta<\pi / 2$. Over the entire measurement domain, turbulence in the wakes of all cases reside in the fifth octant to a large degree. Turbines with $\mathrm{CCW}$ spinning blades (the uniform and checkerboard cases) show a greater tendency to occupy the second octant and turbines with CW rotors occupy the first octant more. For all cases, the residence in the third octant is identically zero. The residence in the seventh octant is low enough $(\sim 0.1 \%)$ to suggest that any residence there is the result of numerical error arising from eigendecomposition.

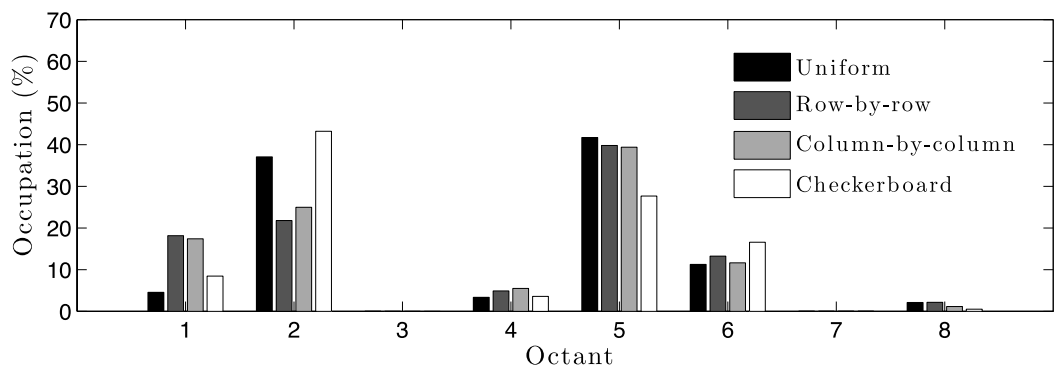

(a)

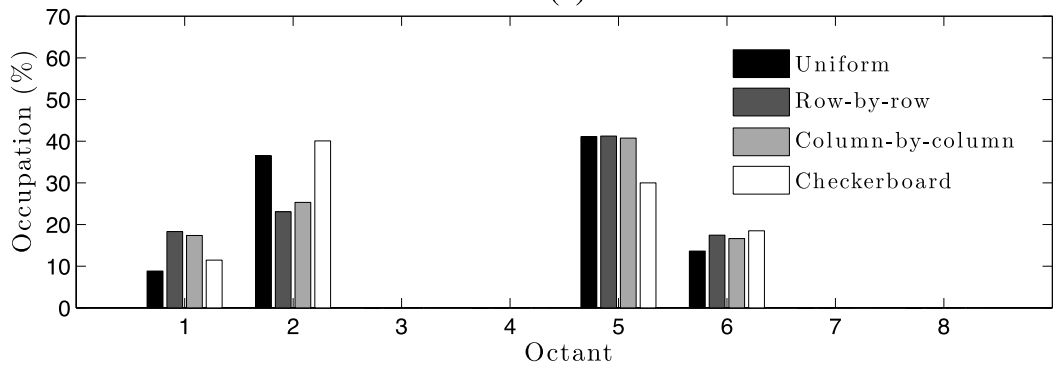

(b)

FIG. 23. Residence of principle axes (and characteristic vector $\vec{r}$ ) in Cartesian octants before and after application of treatment to Euler angles. Data are from the wake areas of the four test cases. (a) Relative occupation of principle axes prior to the application of filters. (b) Relative occupation of principle axes after application of angle filters. 
The residence of the rotated characteristic vector was tallied again after application of the aforementioned filters. Figure 23(b) shows that the filter corrects the octant occupation of $\vec{r}^{\prime}$ by remapping from octants $3,4,7$, and 8 into octants $1,2,5$, and 6 . Differences between test cases are maintained through the filtering process. For example, the octant occupation of $\overrightarrow{r^{\prime}}$ following turbines with CCW rotating blades (uniform and checkerboard cases, black and white bars, respectively) is less in octant 1 and greater in octant 2 than those following CW rotors. This distinction in residence is seen both before and after the filters were applied.

${ }^{1}$ R. B. Cal, J. Lebrón, L. Castillo, H. S. Kang, and C. Meneveau, "Experimental study of the horizontally averaged flow structure in a model wind-turbine array boundary layer," J. Renewable Sustainable Energy 2, 013106 (2010).

${ }^{2}$ N. Hamilton, H.-S. Kang, C. Meneveau, and R. B. Cal, "Statistical analysis of kinetic energy entrainment in a model wind turbine array boundary layer," J. Renewable Sustainable Energy 4, 063105 (2012).

${ }^{3}$ R. J. Barthelmie, O. Rathmann, S. T. Frandsen, K. S. Hansen, E. Politis, J. Prospathopoulos, K. Rados, D. Cabezón, W. Schlez, and J. Phillips, "Modelling and measurements of wakes in large wind farms," J. Phys.: Conf. Ser. 75, 012049 (2007).

${ }^{4}$ A. Crespo, J. Hernández, and S. Frandsen, "Survey of modelling methods for wind turbine wakes and wind farms," Wind Energy 2, 1-24 (1999).

${ }^{5}$ S. Frandsen, R. Barthelmie, S. Pryor, O. Rathmann, S. Larsen, J. Højstrup, and M. Thøgersen, "Analytical modelling of wind speed deficit in large offshore wind farms," Wind Energy 9, 39-53 (2006).

${ }^{6}$ B. Sanderse, "Aerodynamics of wind turbine wakes," Energy Research Center of the Netherlands (ECN), Petten, The Netherlands, Technical Report No. ECN-E-09-016 (2009).

${ }^{7}$ J. Meyers and C. Meneveau, "Optimal turbine spacing in fully developed wind-farm boundary layers," Wind Energy 15, 305-317 (2012).

${ }^{8}$ F. Porté-Agel, Y.-T. Wu, and C.-H. Chen, "A numerical study of the effects of wind direction on turbine wakes and power losses in a large wind farm," Energies 6, 5297-5313 (2013).

${ }^{9}$ R. J. Stevens, D. F. Gayme, and C. Meneveau, "Large eddy simulation studies of the effects of alignment and wind farm length," J. Renewable Sustainable Energy 6, 023105 (2014).

${ }^{10}$ S. T. Frandsen, H. E. Jørgensen, R. Barthelmie, O. Rathmann, J. Badger, K. Hansen, S. Ott, P.-E. Rethore, S. E. Larsen, and L. E Jensen, "The making of a second-generation wind farm efficiency model complex," Wind Energy 12, 445-458 (2009).

${ }^{11}$ C. D. Markfort, W. Zhang, and F. Porté-Agel, "Turbulent flow and scalar transport through and over aligned and staggered wind farms," Journal Turbul. 13, N33 (2012).

${ }^{12}$ H. Snel, "Review of aerodynamics for wind turbines," Wind Energy 6, 203-211 (2003).

${ }^{13}$ M. Calaf, C. Meneveau, and J. Meyers, "Large eddy simulation study of fully developed wind-turbine array boundary layers," Phys. Fluids 22, 015110 (2010).

${ }^{14}$ R. S. Rogallo and P. Moin, "Numerical simulation of turbulent flows," Annu. Rev. Fluid Mech. 16, 99-137 (1984).

15 J. Rotta, "Statistical theory of nonhomogeneous turbulence. ii," Z. Physik 131, 547-572 (1951).

${ }^{16}$ R. Gómez-Elvira, A. Crespo, E. Migoya, F. Manuel, and J. Hernández, "Anisotropy of turbulence in wind turbine wakes," J. Wind Eng. Ind. Aerodyn. 93, 797-814 (2005).

17 A. J. Simonsen and P.-^̊. Krogstad, “Turbulent stress invariant analysis: Clarification of existing terminology,” Phys. Fluids 17, 088103 (2005).

${ }^{18}$ S. C. Tedds, I. Owen, and R. J. Poole, “Near-wake characteristics of a model horizontal axis tidal stream turbine," Renewable Energy 63, 222-235 (2014).

${ }^{19}$ K.-S. Choi and J. L. Lumley, "The return to isotropy of homogeneous turbulence," J. Fluid Mech. 436, 59-84 (2001).

${ }^{20}$ P. Mestayer, "Local isotropy and anisotropy in a high-reynolds-number turbulent boundary layer," J. Fluid Mech. 125, 475-503 (1982).

${ }^{21}$ R. Smalley, S. Leonardi, R. Antonia, L. Djenidi, and P. Orlandi, "Reynolds stress anisotropy of turbulent rough wall layers," Exp. Fluids 33, 31-37 (2002).

${ }^{22}$ S. Leonardi, P. Orlandi, L. Djenidi, and R. A. Antonia, "Structure of turbulent channel flow with square bars on one wall," Int. J. Heat Fluid Flow 25, 384-392 (2004).

${ }^{23}$ W. D. Smyth and J. N. Moum, "Anisotropy of turbulence in stably stratified mixing layers," Phys. Fluids 12, 1343 (2000).

${ }^{24}$ A. Jiménez, A. Crespo, E. Migoya, and J. García, "Advances in large-eddy simulation of a wind turbine wake," J. Phys.: Conf. Ser. 75, 012041 (2007).

${ }^{25}$ I. Ushiyama, T. Shimota, and Y. Miura, “An experimental study of the two-staged wind turbines,” Renewable Energy 9 , 909-912 (1996).

${ }^{26}$ S. N. Jung, T.-S. No, and K.-W. Ryu, "Aerodynamic performance prediction of a $30 \mathrm{~kW}$ counter-rotating wind turbine system," Renewable Energy 30, 631-644 (2005).

${ }^{27}$ S. Lee, H. Kim, E. Son, and S. Lee, "Effects of design parameters on aerodynamic performance of a counter-rotating wind turbine," Renewable Energy 42, 140-144 (2012).

${ }^{28}$ W. Z. Shen, V. A. K. Zakkam, J. N. Sørensen, and K. Appa, “Analysis of counter-rotating wind turbines,” J Phys.: Conf. Ser. 75, 012003 (2007).

${ }^{29}$ W. Yuan, W. Tian, A. Ozbay, and H. Hu, "An experimental study on the effects of relative rotation direction on the wake interferences among tandem wind turbines," Sci. China: Phys., Mech. Astron. 57, 935-949 (2014).

${ }^{30}$ H. S. Kang and C. Meneveau, "Direct mechanical torque sensor for model wind turbines," Meas. Sci. Technol. 21, 105206 (2010).

${ }^{31}$ N. Hamilton, M. Tutkun, and R. B. Cal, "Wind turbine boundary layer arrays for cartesian and staggered configurations: Part II, low-dimensional representations via the proper orthogonal decomposition," Wind Energy (published online 2014).

32 A. J. M. Spencer, “Theory of invariants," Continuum Phys. 1, 239-352 (1971). 
33 J. Diebel, "Representing attitude: Euler angles, unit quaternions, and rotation vectors," Matrix 58, 15-16 (2006).

${ }^{34}$ L. P. Chamorro and F. Porté-Agel, "A wind-tunnel investigation of wind-turbine wakes: Boundary-layer turbulence effects," Boundary Layer Metrol. 132, 129-149 (2009).

${ }^{35}$ N. Hamilton, M. Melius, and R. B. Cal, "Wind turbine boundary layer arrays for Cartesian and staggered configurations: Part I flow field and power measurements," Wind Energy (published online 2014).

${ }^{36}$ L. P. Chamorro, R. E. A. Arndt, and F. Sotiropoulos, "Reynolds number dependence of turbulence statistics in the wake of wind turbines," Wind Energy 15, 733-742 (2012). 